bioRxiv preprint doi: https://doi.org/10.1101/469338; this version posted July 9,2020 . The copyright holder for this preprint (which was not

certified by peer review) is the author/funder, who has granted bioRxiv a license to display the preprint in perpetuity. It is made available under aCC-BY-NC-ND 4.0 International license.

\title{
The stem rust fungus Puccinia graminis f. sp. tritici induces centromeric small RNAs during late infection that direct genome-wide DNA methylation
}

Jana Sperschneider ${ }^{1,2 \#}$, Ashley W. Jones ${ }^{3}$, Jamila Nasim ${ }^{3}$, Bo Xu ${ }^{4}$, Silke Jacques ${ }^{5}$, Narayana M. Upadhyaya ${ }^{2}$, ${\text { Rohit } \text { Mago }^{2} \text {, Melania Figueroa }}^{2}$, Karam B. Singh ${ }^{5,6}$, Eric A. Stone ${ }^{1}$, Benjamin Schwessinger ${ }^{3}$, Ming-Bo Wang $^{2}$, Jennifer M. Taylor ${ }^{2}$, Peter N. Dodds ${ }^{2 \#}$

${ }^{1}$ Biological Data Science Institute, The Australian National University, Canberra, Australia

${ }^{2}$ Black Mountain Science and Innovation Park, CSIRO Agriculture and Food, Canberra, Australia

${ }^{3}$ Research School of Biology, The Australian National University, Acton ACT 2601, Australia

${ }^{4}$ Thermo Fisher Scientific, 5 Caribbean Drive, Scoresby, Australia

${ }^{5}$ Centre for Crop and Disease Management, Department of Environment and Agriculture, Curtin University, Bentley, Australia

${ }^{6}$ Centre for Environment and Life Sciences, CSIRO Agriculture and Food, Perth, Australia

\# Address correspondence to Jana Sperschneider, jana.sperschneider@anu.edu.au and Peter N. Dodds, peter.dodds@csiro.au

\section{Abstract \\ Background}

Silencing of transposable elements (TEs) is essential for maintaining genome stability. Plants use small RNAs (sRNAs) to direct DNA methylation to TEs (RNA-directed DNA methylation; RdDM). Similar mechanisms of epigenetic silencing in the fungal kingdom have remained elusive.

$\underline{\text { Results }}$

We use sRNA sequencing and methylation data to gain insight into epigenetics in the dikaryotic fungus Puccinia graminis f. sp. tritici (Pgt), which causes the devastating stem rust disease on wheat. We use Hi-C data to define the $P g t$ centromeres and show that they are repeat-rich regions $(\sim 250 \mathrm{~kb})$ that are highly diverse in sequence between haplotypes and, like in plants, are enriched for young TEs. DNA cytosine methylation is particularly active at centromeres but also associated with genome-wide control of young TE insertions. Strikingly, over $90 \%$ of $P g t$ sRNAs and several RNAi genes are differentially expressed during infection. Pgt induces waves of functionally diversified sRNAs during infection. The early wave sRNAs are

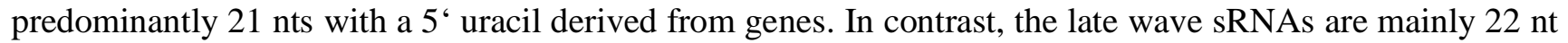
sRNAs with a $5^{\prime}$ adenine and are strongly induced from centromeric regions. TEs that overlap with late wave sRNAs are more likely to be methylated, both inside and outside the centromeres, and methylated TEs exhibit a silencing effect on nearby genes.

Conclusions

We conclude that rust fungi use an epigenetic silencing pathway that resembles RdDM in plants. The Pgt RNAi machinery and sRNAs are under tight temporal control throughout infection and might ensure genome stability during sporulation.

Keywords: small RNA, methylation, stem rust, Puccinia graminis f. sp. tritici, fungal pathogen, centromeres 


\section{Background}

Epigenetic regulation controls transcription through formation of transcriptionally inactive chromatin (heterochromatin) and are mediated by interactions between small RNAs (sRNAs), DNA methylation and/or repressive histone modifications. In plants, sRNAs are predominantly in the size range of 20-24 nt and can be divided into two classes: 1) small interfering RNAs (siRNAs) processed by Dicer proteins from long double-stranded RNA (dsRNA), and 2) microRNAs (miRNAs) processed from stem-loop regions of singlestranded primary RNAs [1]. Both miRNAs and siRNAs are bound to argonaute (AGO) proteins to induce silencing of targets by base-pairing interactions and complementarity [2].

Heterochromatin plays both regulatory and structural roles. Heterochromatin regulates gene transcription, but also ensures proper chromosome segregation during cell division at centromeres and genome stability through regulation of transposable elements (TEs) [3]. Epigenetic silencing in repetitive genome regions is a key mechanism to prevent proliferation of TEs. In fungi and plants, DNA cytosine methylation (5methylcytosine; $5 \mathrm{mC}$ ) is found mainly in transposable elements and other repeated DNA [4, 5]. In plants, RNA-directed DNA methylation (RdDM) is the major sRNA-mediated epigenetic pathway and functions in maintaining genome stability through transposon control, pathogen defence and stress responses, intercellular communication and germ cell specification [6]. RdDM uses sRNAs to trigger DNA cytosine methylation at homologous DNA sequences in the genome [7]. These nuclear-localized heterochromatic sRNAs are the most abundant sRNAs in plants, predominantly 24 nucleotides (nts) in length, derived from intergenic or repetitive regions and associated with the argonaute 4 (AGO4) clade to regulate epigenetic silencing. Adenine is the most common 5' base of AGO4-bound 24 nt sRNAs in Arabidopsis [8].

Unlike the extensively studied RdDM pathway in plants [9], the mechanisms of epigenetic silencing in the diverse fungal kingdom have remained elusive [10]. The RNAi machinery of the fission yeast Schizosaccharomyces pombe and the filamentous fungus Neurospora crassa are thus far the best-studied non-pathogenic model species [11]. In fission yeast, RNAi components participate in heterochromatin formation through histone $\mathrm{H} 3 \mathrm{~K} 9$ modifications at centromeres, the mating type interval and the subtelomeric regions [12,13]. DNA cytosine methylation is absent in the model yeasts $S$. pombe and S. cerevisiae [14]. In Neurospora crassa, RNAi components are involved in quelling and meiotic silencing by unpaired DNA (MSUD), but not in heterochromatin formation. Quelling is an RNAi-related gene-silencing mechanism in Neurospora that is induced by repetitive transgenic sequences and occurs in the vegetative growth stage to control transposons [15]. Outside the model fungal species, very little is known about the interplay between sRNAs and epigenetic silencing, particularly in highly repetitive fungal pathogen genomes that need to inactivate TEs. Unlike plants, fungi lack canonical gene-body methylation but in line with plants, $5 \mathrm{mC}$ is abundant in repetitive DNA and transposons across fungal species [4]. How RNAi contributes to epigenetic silencing in other fungi has thus far largely remained unexplored. In the ascomycete Magnaporthe oryzae, a plant pathogen, 18-23 nt sRNAs are produced from repetitive elements and are implicated in TE regulation in vegetative tissue, whereas 28-35 nt sRNAs mapping to transfer RNA (tRNA) loci are enriched in the appressoria [16]. However, a correlation between sRNAs and epigenetic silencing has not been shown in $M$. oryzae. In the white-rot basidiomycete fungus Pleurotus ostreatus, TE silencing is associated with $21 \mathrm{nt}$ sRNAs and DNA methylation [17]. RNAi has been suggested as a key determinant of longer centromeres in the human fungal pathogen Cryptococcus and as a suppressor of centromeric retrotransposons to ensure genome stability [18].

The basidiomycete fungus Puccinia graminis f. sp. tritici (Pgt) is a plant pathogen that causes wheat stem rust disease, resulting in devastating crop losses [19]. Pgt is a dikaryotic fungus that contains two distinct haploid nuclei. During the asexual infection phase on a cereal host, Pgt produces single-celled dikaryotic urediniospores that germinate on the leaf surface [20, 21]. Subsequently, appressoria form and penetration occurs through stomata with subsequent development of specialized infection structures called haustoria by around 2 days. Haustoria enable nutrient uptake as well as the delivery of secreted pathogen proteins called effectors into the host plant cell [22]. The start of urediniospore production occurs at approximately 6-7 days post infection (dpi) and urediniospore pustules typically erupt through the leaf or stem surface (sporulation) 
after 8-10 dpi [20]. In the poplar rusts, intense cell division activity has been observed in the sporulation area [23].

Chromosome-scale genome assemblies offer the opportunity to investigate the structural organisation of the genome including localization of centromeres, transposable elements (TEs), DNA methylation, sRNAs and how this links to their function. Recently, the chromosome-scale assembly of Pgt 21-0 has become available [24]. This assembly is fully phased with 18 chromosome pseudomolecules for each of the two haplotypes derived from the two haploid nuclei. Whilst substantial time-course transcriptomic resources have been generated for Pgt [25-27], how it utilizes RNAi and epigenetic silencing during the infection cycle has thus far been unknown. Here, we bring together Hi-C data, methylation data and sRNA/transcriptome sequencing data to uncover fundamental characteristics of the stem rust RNAi machinery, DNA methylation and the first-time characterization of rust centromeres.

\section{Results}

\section{Pgt centromeres are highly repetitive sequences with little sequence conservation between haplotypes}

We used chromatin conformation capture assay data (Hi-C) from Pgt 21-0 [24] to pinpoint the location of the $P g t$ centromeres, the first-time characterization of rust fungi centromeres. Fungal centromeres give rise to a distinct outwards-spreading shape in a Hi-C contact map [28], as seen in the contact maps for individual chromosomes (Supplementary Figures S1 and S2). Because the centromeres of each chromosome tend to cluster in the three-dimensional space of the nucleus, this region also shows physical association between chromosomes visible as distinct cross-shapes in the whole haplotype Hi-C contact map (Figure 1A). We selected the midpoint of the outwards-spreading shape in the Hi-C contact maps of each chromosome as the putative centre of each centromere. For example, Pgt chromosome 1A has a suggested centromere centre around position $2.36 \mathrm{MB}$ and the surrounding region shares strong $\mathrm{Hi}-\mathrm{C}$ links with the putative centromeres on other chromosomes (Figure 1A and Supplementary Figure S3). To add further support to the centromeric regions and their lengths, we plotted the density of expressed genes, RNA-seq transcription levels at late infection and in germinated spores as well as the coverage of repetitive elements on the chromosomes. This shows that the regions around the putative centromere centres indicated by the $\mathrm{Hi}-\mathrm{C}$ data are transcriptionally silent, gene-poor and repeat-rich regions (Figures 1B and 2, Supplementary Figure S4). We selected putative centromere boundaries by inspecting the lengths of these transcriptionally silent, gene-poor regions for each chromosome (Figure 2). Centromeres appear to span between $100 \mathrm{~kb}$ to $340 \mathrm{~kb}$ ( $253 \mathrm{~kb}$ on average), with only a slight decrease in GC content for most chromosomes compared to the rest of the chromosome

(Table

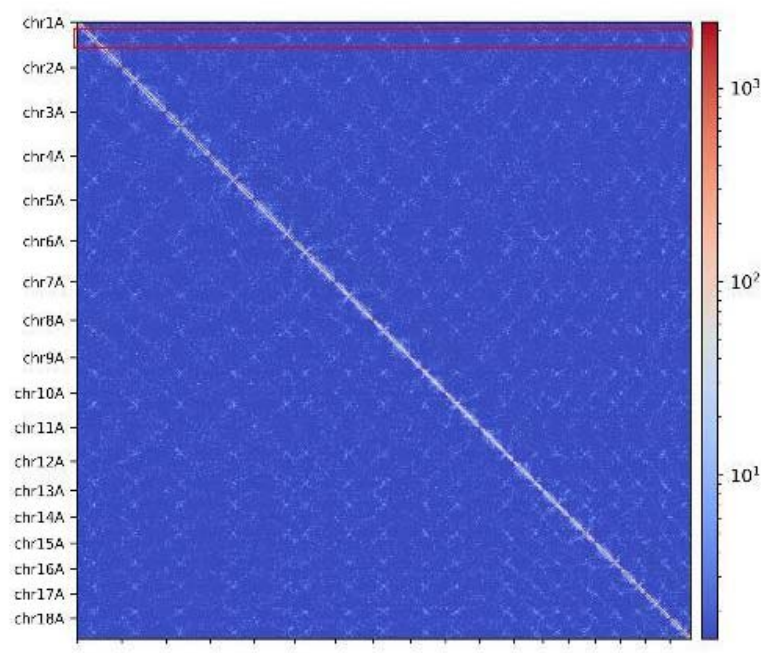

(A)

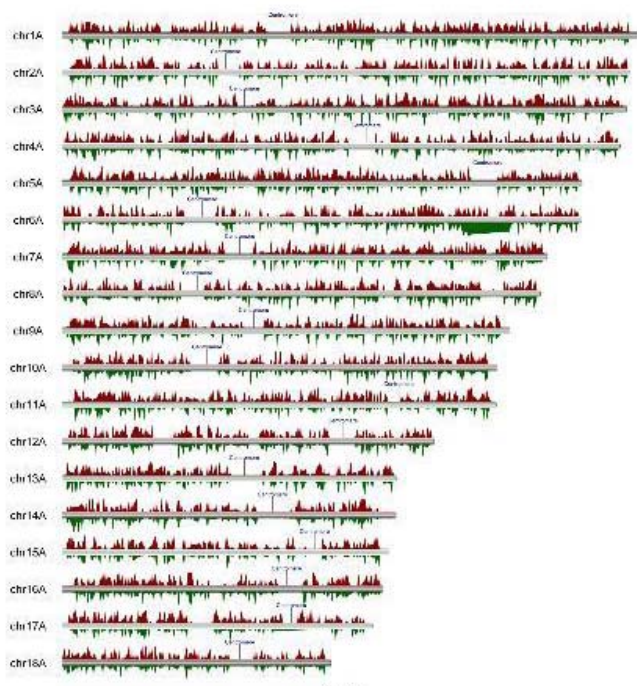

(B) 
bioRxiv preprint doi: https://doi.org/10.1101/469338; this version posted July 9, 2020. The copyright holder for this preprint (which was not certified by peer review) is the author/funder, who has granted bioRxiv a license to display the preprint in perpetuity. It is made available under

Figure 1: Hi-C contact map shows the location of the Pgt centromeres. (A) A Hi-C contact map of the 18 chromosomes in haplotype A shows the position of the centromeres as cross-like shapes, highlighted with a red rectangle. (B) The positions of the centromeres in haplotype A as indicated by the Hi-C contact map are in transcriptionally silent genomic regions. Reads per million (RPM) for the late infection (7 dpi) and germinated spores RNAseq samples are shown in red and green, respectively (10 kb windows, RPM from 0-100 are shown for clarity).

$\operatorname{chr} 1 \mathrm{~A}$
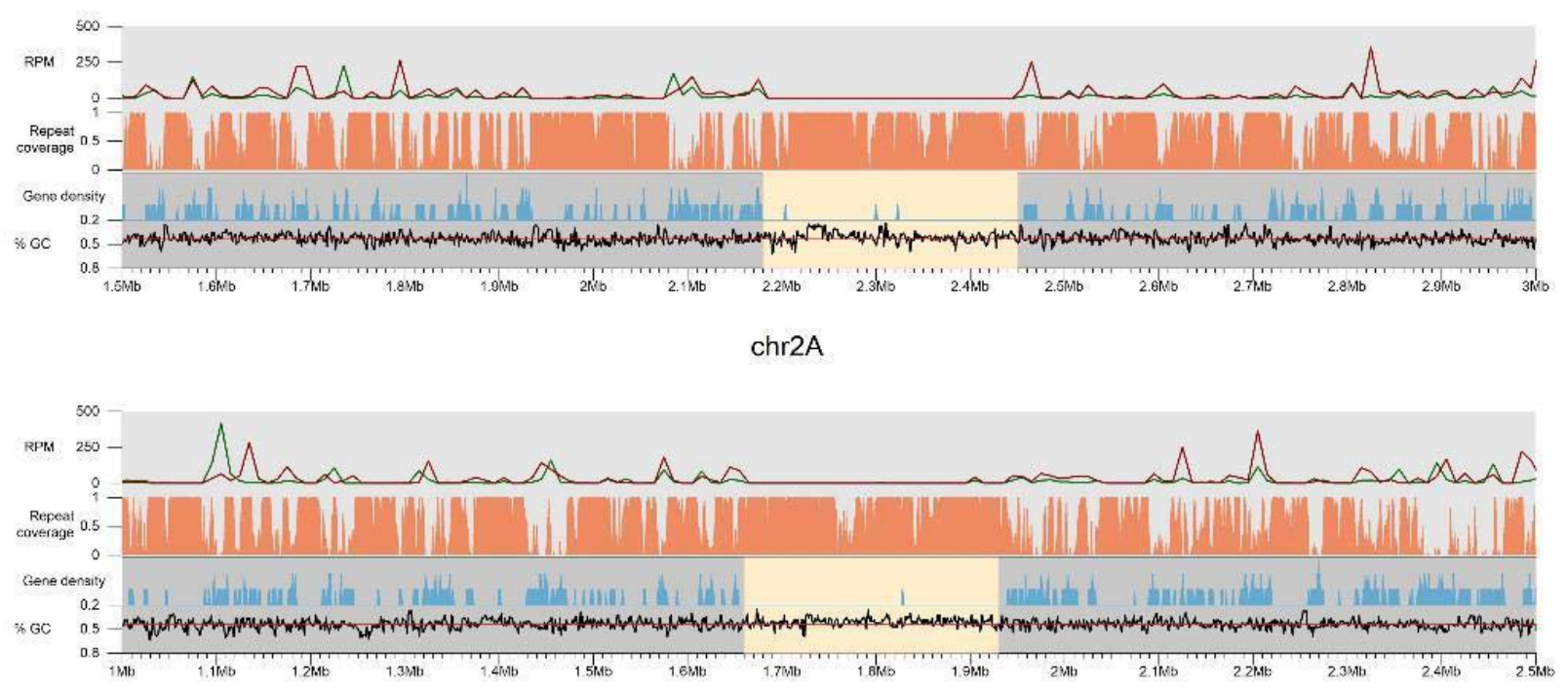

Figure 2: Pgt centromeric regions for two selected chromosomes. Karyoplots of the centromeric regions of Pgt chromosomes $1 \mathrm{~A}$ and $2 \mathrm{~A}$. The density of expressed genes and the coverage of repetitive elements are shown as well as the GC content (1 kb windows). Reads per million (RPM) for the late infection (7 dpi) and germinated spores RNAseq samples are shown as red and green lines, respectively (10 kb windows). Centromeric regions are highlighted with yellow boxes.

Table 1: Genomic coordinates, lengths and GC content of the centromeric regions for each Pgt chromosome of the A and B haplotypes.

\begin{tabular}{r|r|r|r} 
Chromosome & Centromeric region & Centromere length & $\begin{array}{r}\text { GC content } \\
\text { (centromere/non-centromere) }\end{array}$ \\
\hline $1 \mathrm{~A}$ & $2.18-2.45 \mathrm{MB}$ & $270 \mathrm{~kb}$ & $40.6 \% / 43.7 \%$ \\
$1 \mathrm{~B}$ & $2.44-2.72 \mathrm{MB}$ & $280 \mathrm{~kb}$ & $41.9 \% / 43.6 \%$ \\
$2 \mathrm{~A}$ & $1.66-1.93 \mathrm{MB}$ & $270 \mathrm{~kb}$ & $40.9 \% / 43.8 \%$ \\
2B & $1.56-1.83 \mathrm{MB}$ & $270 \mathrm{~kb}$ & $41.8 \% / 43.7 \%$ \\
$3 \mathrm{~A}$ & $1.87-2.09 \mathrm{MB}$ & $220 \mathrm{~kb}$ & $41.9 \% / 43.8 \%$ \\
3B & $1.92-2.26 \mathrm{MB}$ & $340 \mathrm{~kb}$ & $43.4 \% / 43.3 \%$ \\
$4 \mathrm{~A}$ & $3.06-3.34 \mathrm{MB}$ & $280 \mathrm{~kb}$ & $42.1 \% / 43.7 \%$ \\
$4 \mathrm{~B}$ & $3.33-3.64 \mathrm{MB}$ & $310 \mathrm{~kb}$ & $42.6 \% / 43.5 \%$ \\
$5 \mathrm{~A}$ & $4.38-4.65 \mathrm{MB}$ & $270 \mathrm{~kb}$ & $42.3 \% / 43.8 \%$ \\
$5 \mathrm{~B}$ & $6.01-6.23 \mathrm{MB}$ & $220 \mathrm{~kb}$ & $42.4 \% / 43.9 \%$ \\
$6 \mathrm{~A}$ & $1.30-1.62 \mathrm{MB}$ & $320 \mathrm{~kb}$ & $43.3 \% / 43.5 \%$ \\
6B & $1.15-1.49 \mathrm{MB}$ & $340 \mathrm{~kb}$ & $42.8 \% / 43.2 \%$ \\
$7 \mathrm{~A}$ & $1.74-2.04 \mathrm{MB}$ & $300 \mathrm{~kb}$ & $41.9 \% / 43.6 \%$ \\
$7 \mathrm{~B}$ & $1.94-2.18 \mathrm{MB}$ & $240 \mathrm{~kb}$ & $41.1 \% / 43.4 \%$ \\
$8 \mathrm{~A}$ & $1.24-1.52 \mathrm{MB}$ & $280 \mathrm{~kb}$ & $41.6 \% / 43.6 \%$ \\
8B & $1.28-1.55 \mathrm{MB}$ & $270 \mathrm{~kb}$ & $42.5 \% / 43.9 \%$ \\
$9 \mathrm{~A}$ & $1.93-2.16 \mathrm{MB}$ & $230 \mathrm{~kb}$ & $41.9 \% / 44.0 \%$ \\
9B & $2.12-2.36 \mathrm{MB}$ & $240 \mathrm{~kb}$ & $41.9 \% / 43.7 \%$ \\
$10 \mathrm{~A}$ & $1.37-1.66 \mathrm{MB}$ & $290 \mathrm{~kb}$ & $42.8 \% / 43.7 \%$ \\
$10 \mathrm{~B}$ & $1.98-2.25 \mathrm{MB}$ & $270 \mathrm{~kb}$ & $42.9 \% / 43.4 \%$ \\
$11 \mathrm{~A}$ & $3.5-3.6 \mathrm{MB}$ & $100 \mathrm{~kb}$ & $40.5 \% / 43.8 \%$ \\
$11 \mathrm{~B}$ & $3.82-3.93 \mathrm{MB}$ & $110 \mathrm{~kb}$ & $40.5 \% / 43.7 \%$
\end{tabular}


bioRxiv preprint doi: https://doi.org/10.1101/469338; this version posted July 9, 2020. The copyright holder for this preprint (which was not certified by peer review) is the author/funder, who has granted bioRxiv a license to display the preprint in perpetuity. It is made available under aCC-BY-NC-ND 4.0 International license.

39

We then aligned the two haplotype chromosomes. Interestingly, some chromosomes share regions of macrosynteny (conserved regions $>20 \mathrm{~kb}$ ) in their centromeres whereas others do not. For example, chromosomes $1 \mathrm{~A}$ and $1 \mathrm{~B}$ show no to very low sequence identity in the centromeric region, as opposed to the remainder of the chromosome (Figure 3A). In contrast, chromosomes 2A and 2B share macro-synteny in the centromeric region (Figure $3 \mathrm{~B}$ ). We then used pairwise $k$-mer distance estimation to compare centromeric regions and non-centromeric regions for all chromosomes. Clustering analysis showed a large distance between the centromeric regions and non-centromeric regions (Figure 3C). For the non-centromeric regions, the two homologous chromosomes all grouped into closely related pairs with similar distances for all 18 chromosome pairs. In contrast, while most (14/18) centromeric regions grouped by chromosome pairs, the difference between them varied, with some very closely related (e.g. on chromosomes $2 \mathrm{~A}$ and $2 \mathrm{~B}$ ) and others quite divergent (e.g. chromosomes $1 \mathrm{~A}$ and 1B). Others showed unexpected groupings. For example, the centromeres of chromosomes $18 \mathrm{~A}$ and $15 \mathrm{~B}$ are more closely related to each other than to the centromeres of the corresponding chromosomes 18B and 15A (Figure 3C). Overall, the centromeric regions of $P g t$ are highly variable and unexpectedly, most of them are also highly divergent between haplotypes.
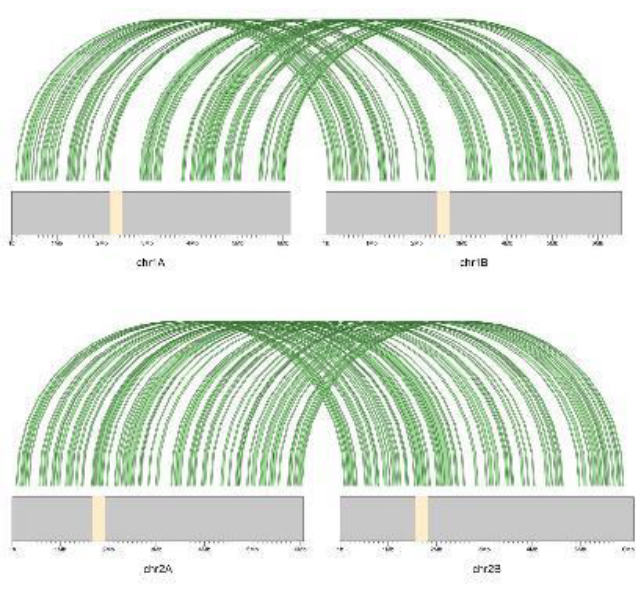

(A)

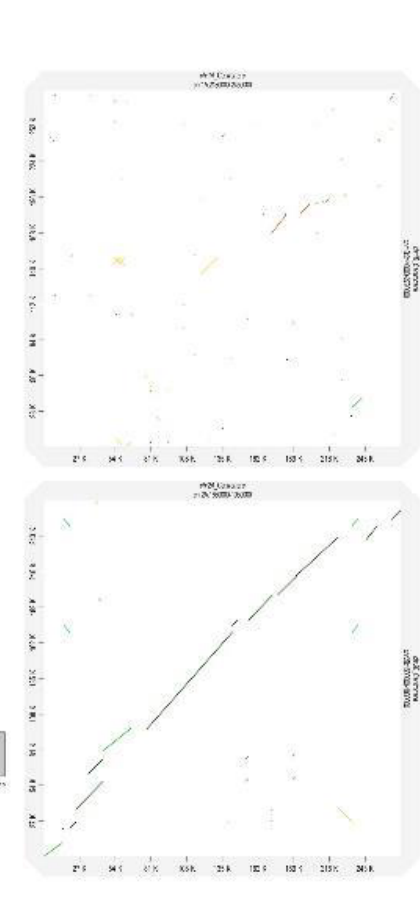

(B)

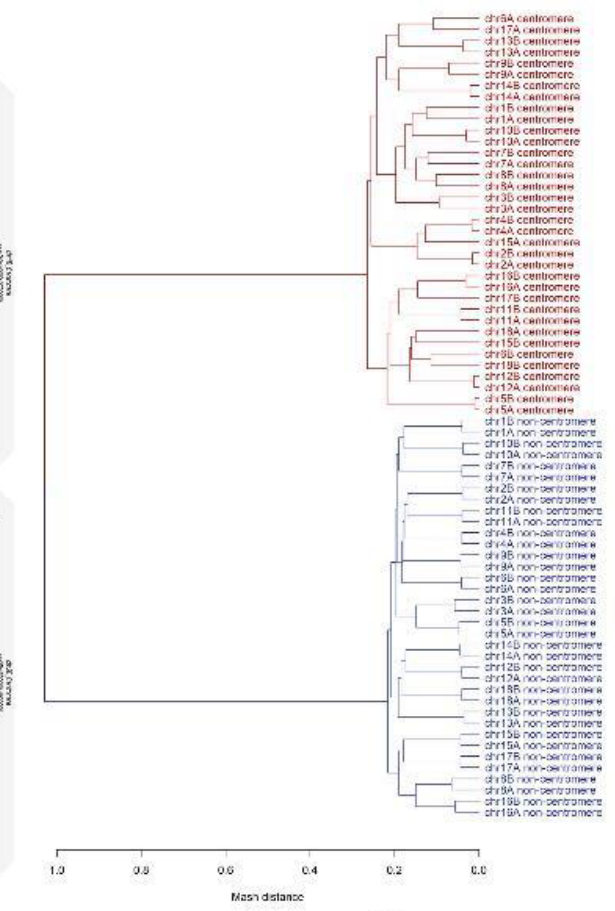

(C)

Figure 3: Synteny and sequence similarity of the Pgt centromeres. (A) Regions of macrosynteny (> $20 \mathrm{kbp}$ ) between the haplotypes of chromosomes 1 and 2. For chromosome 1, the centromeric regions show no conservation 
whereas on chromosome 2 the centromeric regions are conserved as confirmed by (B) genomic dot plot alignments of the centromeric regions. (C) Clustering of $k$-mer distance estimations between centromeric and non-centromeric regions. The non-centromeric regions cluster as expected according to haplotypes. In contrast, the centromeric regions are highly divergent, even between haplotypes.

\section{Young transposable elements accumulate in the highly repetitive Pgt centromeric regions}

We then assessed the repetitive element coverage of the Pgt chromosomes and their centromeres. All 36 $(2 * 18)$ Pgt centromere regions have higher coverage of repetitive elements than the non-centromeric regions (Figure 4A). Repetitive elements cover 75\%-96\% of the bases in the Pgt centromeres compared to only $52 \%-62 \%$ of the non-centromeric regions on the chromosomes. The repeat types with the highest sequence coverage in the centromeric regions vary considerably between the chromosomes. Most centromeres are enriched for LTR Gypsy retrotransposons (17\%-56\%) compared to non-centromeric regions (11-17\%), although this is also the most abundant repeat family outside the centromeres (Figure 4B). However, several centromeres show a high coverage of repeat families that are of low abundance outside the centromeric regions. For example, DNA transposons of the superfamily CACTA are highly abundant in the centromere of chromosome 17A (33\% coverage), while the centromere of chromosome 11B is enriched for LTR Copia retrotransposons (35\% coverage). Again, these patterns are not always shared between centromeres within a chromosome pair.

To determine whether the age of TEs affects their distribution, we used the nucleotide identity of each TE to the consensus sequence of the family as a proxy for the relative age of TE insertion. Most TEs in the Pgt genome have $>70 \%$ identity to the consensus, however the centromeres are enriched for young TEs (defined as having $>90 \%$ identity, Figure 4C). In the centromeres, $28.3 \%$ of repeats with family identity information are young TEs compared to $18.8 \%$ outside the centromeres. Taken together, the centromeres are highly repetitive regions in the Pgt genome that are enriched for young TEs, similarly to Arabidopsis where the majority of young repeats are found in pericentromeric domains [29]. 
bioRxiv preprint doi: https://doi.org/10.1101/469338; this version posted July 9, 2020. The copyright holder for this preprint (which was not certified by peer review) is the author/funder, who has granted bioRxiv a license to display the preprint in perpetuity. It is made available under aCC-BY-NC-ND 4.0 International license.

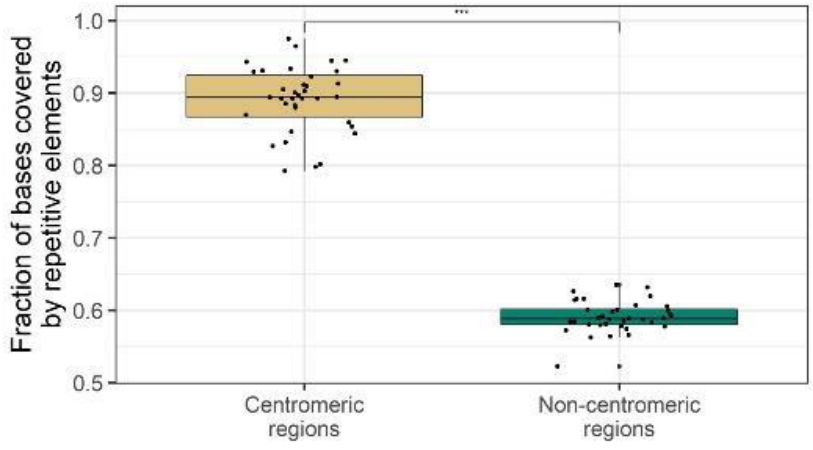

(A)

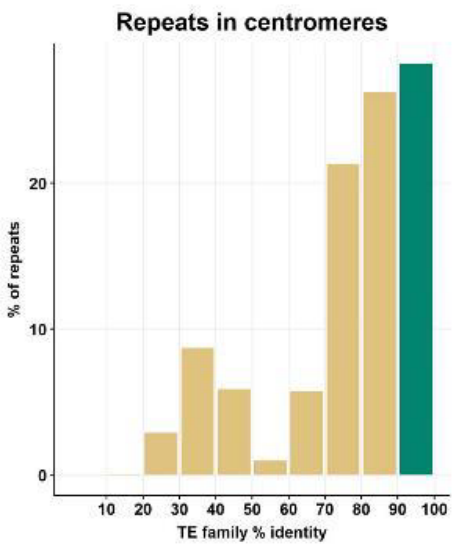

(C)

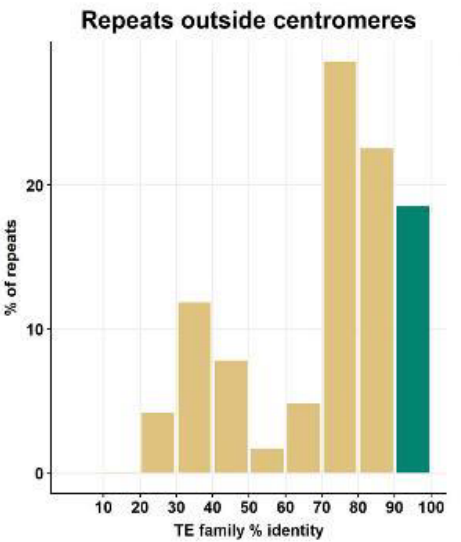

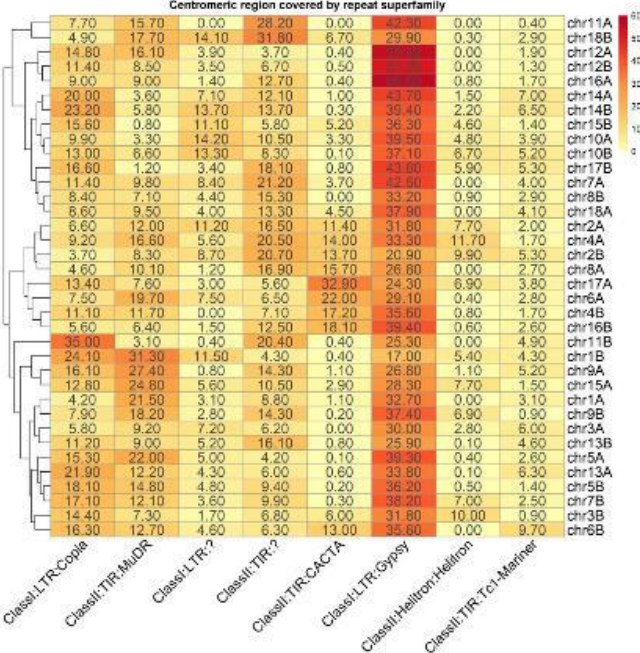

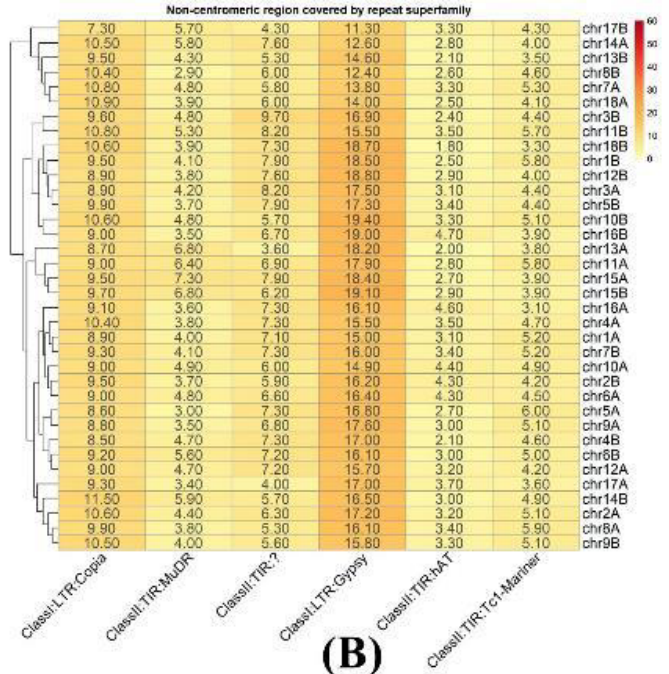

Figure 4: Properties of repetitive elements in the centromeres. (A) The repetitive element coverage of centromeric regions is significantly higher than the repetitive element coverage of non-centromeric regions for all the Pgt chromosomes. (B) Percent of bases that are covered by repetitive elements of a particular class. The centromeric regions vary considerably in the types of repeats they harbour, even between haplotypes. (C) The centromeres have a large proportion of young transposable element (TE) insertions compared to the non-centromeric regions.

\section{Pgt centromeres are heavily $\mathbf{5 m C}$-methylated at genomic $\mathbf{C G}$ sites}

We used Nanopore sequencing to detect DNA methylation in genomic DNA of Pgt during two distinct infection stages: 1) germination of spores and 2) late infection stage of wheat when sporulation starts (7 dpi). The Nanopore signal distinguishes 5-methylcytosine $(5 \mathrm{mC})$ from unmethylated cytosine and N6methyladenine (6mA) from unmethylated adenine [30]. Methylated sites were defined as nucleotide positions where more than $50 \%$ of sequence reads showed the presence of a modified residue. We found that the occurrence of methylated cytosine residues was substantially higher in CG dinucleotide and CCG trinucleotides than in other di- and trinucleotide contexts, similar to cytosine methylation patterns in plants. The proportion of both CG and CCG methylation sites was significantly higher in centromeric regions $(18 \%$ and $7.5 \%$ ) than in non-centromeric regions $(7.5 \%$ and $3.6 \%)$. Levels of $6 \mathrm{~mA}$ methylation were low both inside and outside the centromeres with no substantial difference between dinucleotide contexts (Figure 5A). The frequency of methylation at methylated CG dinucleotide sites (i.e. \% of reads from a site that show base modifications) are also higher for sites that occur in the centromeres than for those outside the centromeres, but very similar between germinated spores and late infection (Figure 5B). Taken together, Pgt has a strong preference for genomic $\mathrm{CG}(\mathrm{CpG})$ methylation and centromeres are heavily CG-methylated genomic regions. 


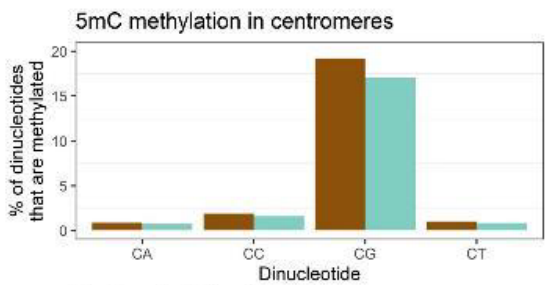
aCC-BY-NC-ND 4.0 International license.
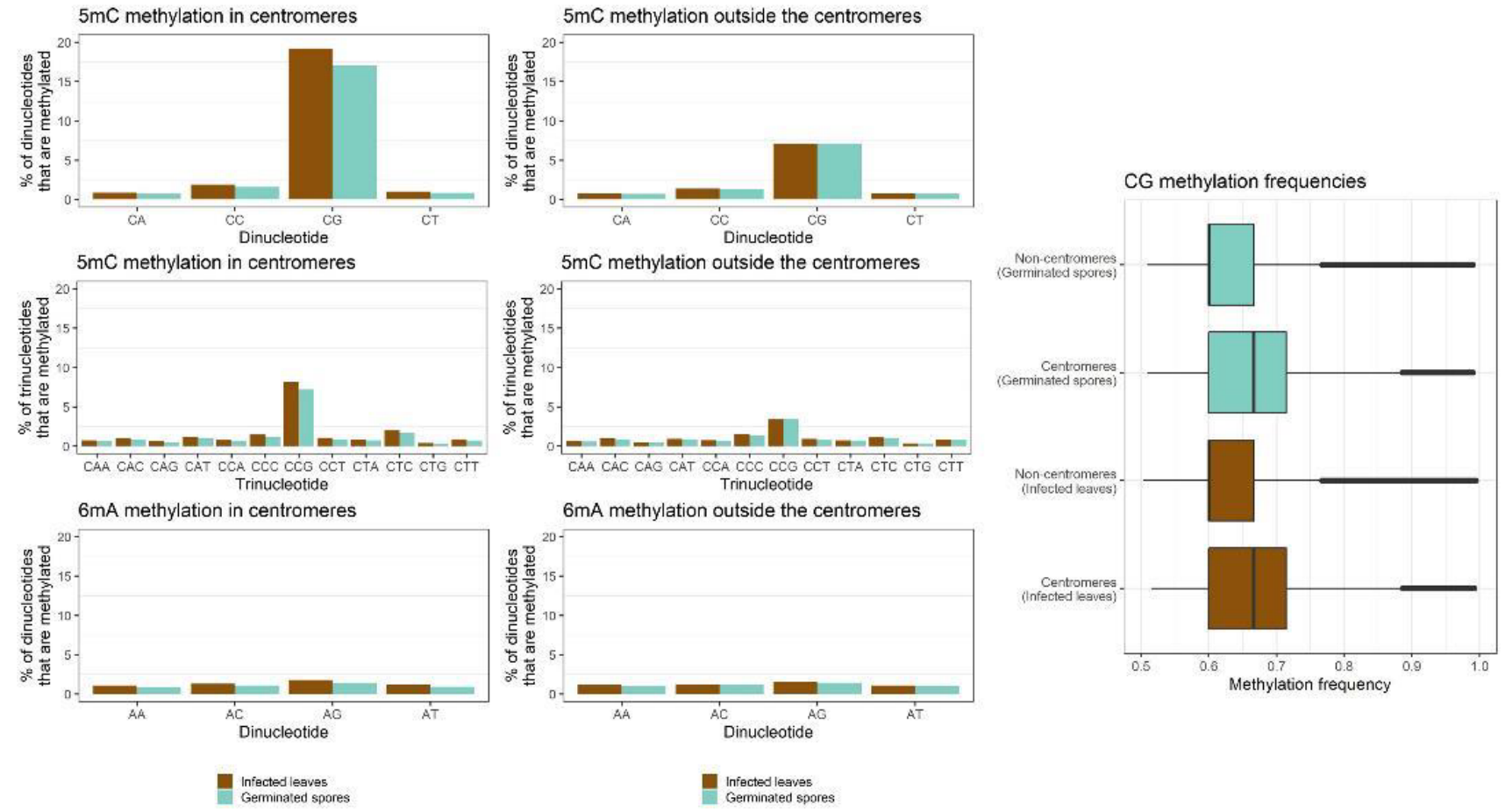

(A)

(B)

Figure 5: Methylation site preferences in Pgt. (A) Proportions of Pgt dinucleotides and trinucleotides that are methylated in the centromeres and outside the centromeres. CG dinucleotides are highly enriched for $5 \mathrm{mC}$ methylation. For the trinucleotides, CCG is enriched for $5 \mathrm{mC}$ methylation. We observed very low levels of $6 \mathrm{~mA}$ methylation. The centromeres are heavily methylated compared to the non-centromeric regions. Slightly higher levels of $5 \mathrm{mC}$ methylation are seen in infected leaves compared to germinated spores in centromeres. (B) Box plots showing methylation frequency distribution of CG methylation sites. Centromeres show higher methylation frequencies than non-centromeric regions.

\section{CG methylation is associated with silencing of young TE insertions both inside and outside of centromeres}

CG methylation is strongly associated with repetitive regions in both germinated spores and late infection, with $89.3 \%$ and $88.3 \%$ of methylation sites overlapping with TEs, respectively. $12.2 \%$ of all Pgt TEs are methylated in germinated spores and $12.2 \%$ in late infection. $47 \%$ of methylated TEs show methylation in both conditions. A higher percentage of TEs in centromeric regions are methylated $(21.4 \%$ and $17.2 \%$ in infected leaves and germinated spores, respectively) than TEs in non-centromeric regions (11.8\% and $12 \%$ in infected leaves and germinated spores, respectively). We did not observe significant differences in TE family distributions for TEs that are methylated only in germinated spores or only in infected leaves (data not shown).

CG methylation is strongly associated with young TEs (> 90\% identity), not only inside the centromeric regions but even more so outside the centromeres. $21.2 \%$ and $25.1 \%$ of young TEs in the centromeres are methylated in germinates spores and in late infection, respectively (Figure 6). Whilst the centromeres are highly methylated genomic regions and preferentially harbour young TE elements (Figure 4C), young TEs that occur outside the centromeres are also heavily methylated. $23.6 \%$ of young TEs outside the centromeres are methylated both in germinated spores and in infected leaves (Figure 6). This suggests that Pgt employs a mechanism that maintains silencing of young TEs not only in the centromeres but also through targeting of their homologous sequences outside the centromeres. We hypothesized that Pgt might employ sRNAs to direct DNA methylation to young TEs outside the centromeres. 


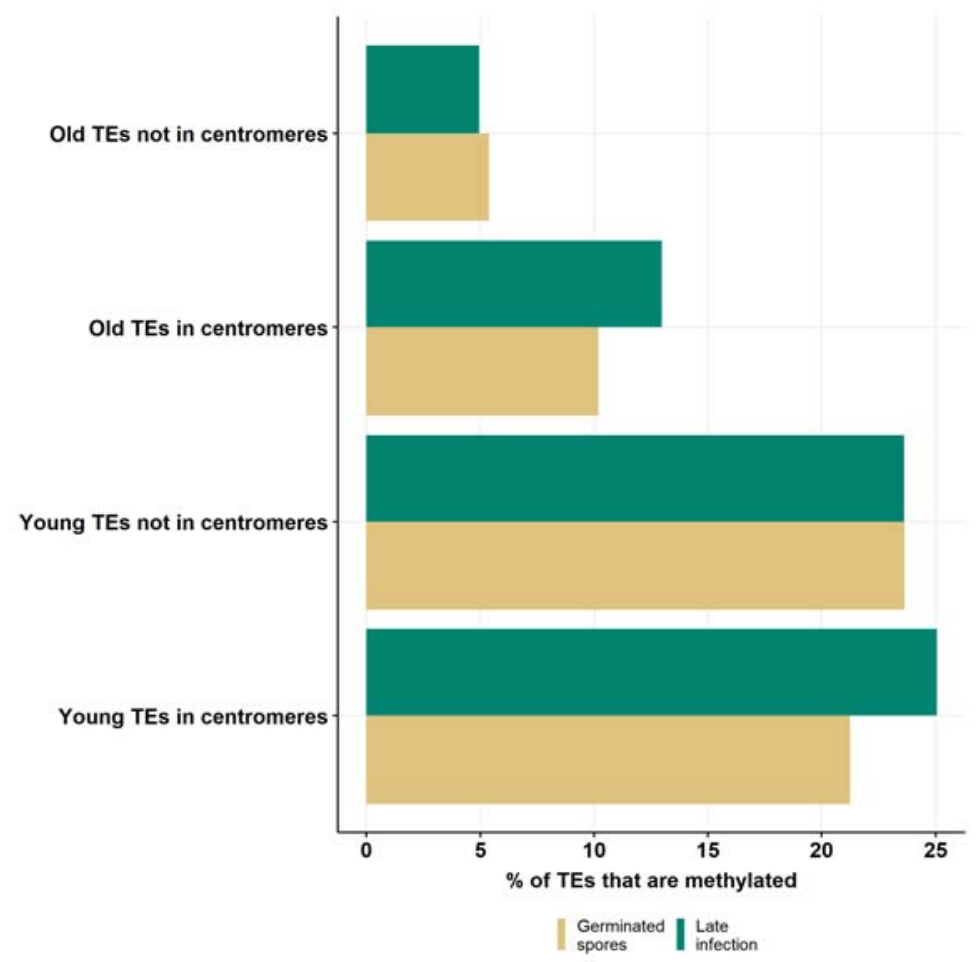

Figure 6: Proportions of young and old Pgt TEs that are methylated in the centromeres and outside the centromeres. Both inside and outside the centromeres, young TEs (> 90\% family identity) are preferentially targeted by CG methylation.

\section{Pgt induces early and late waves of sRNAs with opposing profiles}

To assess the role of the RNAi machinery in methylation, we performed sRNA-sequencing on germinated spores, uninfected wheat and infected wheat at $3 \mathrm{dpi}, 5 \mathrm{dpi}$ and $7 \mathrm{dpi}$. Adapter-trimmed and tRNA/rRNAfiltered sRNA reads were first mapped to the wheat and $P g t$ genomes. Strikingly, the read alignment rates show a strong presence of $P g t$ sRNAs in the late infection sample ( $7 \mathrm{dpi}$, Table 2$)$. The mapping rates to rust in early infection ( $3 \mathrm{dpi}$ and $5 \mathrm{dpi}$ ) are low at $5.25 \%$ and $5.37 \%$, respectively, but increase drastically to $50.18 \%$ in late infection ( $7 \mathrm{dpi}$ ). In contrast, $\sim 67 \%$ of sRNA reads map to the wheat genome in early infection and in late infection the sRNA mapping rate to wheat decreases to $30.3 \%$.

Table 2: Small RNA read mapping rates to the wheat and rust genomes.

\begin{tabular}{lrrr}
\hline Sample & Number of reads & Mapped to Pgt & Mapped to wheat \\
\hline Germinated spores & $27,536,477$ & $55.93 \%$ & $0.73 \%$ \\
\hline Uninfected wheat & $2,353,359$ & $3.56 \%$ & $70.34 \%$ \\
\hline Infected wheat 3dpi & $3,040,002$ & $5.25 \%$ & $67.56 \%$ \\
\hline Infected wheat 5dpi & $2,914,397$ & $5.37 \%$ & $66.98 \%$ \\
\hline Infected wheat 7dpi & $5,815,521$ & $50.18 \%$ & $30.3 \%$ \\
\hline
\end{tabular}

We predicted 7,304 Pgt sRNA loci (7,299 siRNAs and 5 miRNAs) and 411 wheat sRNA loci (360 siRNAs and 51 miRNAs) (Supplementary Files S1-S4). For each predicted sRNA locus, we obtained the most abundant sRNA. For predicted miRNA loci, this will generally be the functional mature miRNA. The read length distributions of rust and wheat sRNAs show different patterns and distinct peaks of abundance (Figure 7). The Pgt-derived sRNAs are predominantly 20, 21 or $22 \mathrm{nts}$ in length. This is true for both for the single most abundant sRNA in each locus as well as for the total sRNA reads derived from each locus (Figure 7). There are two distinct peaks at $21 \mathrm{nt}$ and $24 \mathrm{nt}$ for the wheat sRNAs, as is expected for plant 

aCC-BY-NC-ND 4.0 International license.

sRNAs. Most predicted wheat miRNAs are $21 \mathrm{nt}$ and have a 5' uracil (71.4\%) while the wheat siRNAs are mostly either $21 \mathrm{nt}$ with a 5' uracil or $24 \mathrm{nt}$ with a 5' adenine (Table 3). The two distinct peaks at 21 and 24 nts with their corresponding $5^{\prime}$ nucleotide preferences support the predicted presence of both miRNAs and siRNAs in the wheat sRNA set and the 24 nt wheat siRNAs are likely involved in RdDM [8, 31]. However, two distinct classes of siRNAs also appear to be present in Pgt based on 5' nucleotide preference, although differing in size to the wheat siRNAs. Pgt siRNAs of length 20-21 nts have a strong preference for a 5 " uracil $(\sim 6 \%)$, whereas $54 \%$ of the 22 nt $P g t$ siRNAs have a $5^{*}$ adenine, suggesting functional diversification.

Table 3: Predicted miRNAs and siRNAs in Pgt and wheat and their properties.

\begin{tabular}{lrrrr}
\hline & Pgt siRNAs & Pgt miRNAs & Wheat siRNAs & Wheat miRNAs \\
\hline \# of sRNAs & 7,299 & 5 & 360 & 51 \\
\hline $20 \mathrm{nts}(5, \mathrm{~A} \mid 5, \mathrm{U})$ & $26.5 \%(16 \% \mid 76 \%)$ & $0 \%$ & $8.3 \%(17 \% \mid 73 \%)$ & $13.7 \%(0 \% \mid 100 \%)$ \\
$21 \mathrm{nts}(5, \mathrm{~A} \mid 5, \mathrm{U})$ & $34.7 \%(19 \% \mid 77 \%)$ & $0 \%$ & $47.2 \%(21 \% \mid 46 \%)$ & $68.6 \%(11 \% \mid 71 \%)$ \\
$22 \mathrm{nts}(5, \mathrm{~A} \mid 5, \mathrm{U})$ & $31.8 \%(54 \% \mid 44 \%)$ & $100 \%(60 \% \mid 40 \%)$ & $14.7 \%(30 \% \mid 60 \%)$ & $15.7 \%(0 \% \mid 88 \%)$ \\
$24 \mathrm{nts}(5, \mathrm{~A} \mid 5, \mathrm{U})$ & $0 \%$ & $0 \%$ & $25.6 \%(45 \% \mid 23 \%)$ & $0 \%$ \\
\hline
\end{tabular}

Next, we assessed the differential expression of Pgt sRNAs at the start of infection (germinated spores), during early infection ( $3 \mathrm{dpi}$ and $5 \mathrm{dpi}$ ) and during late infection when sporulation begins ( $7 \mathrm{dpi})$. We detected no differential expression of $P g t$ sRNAs between 3 dpi and 5 dpi, likely due to the low number of mapped reads (Table 2, Figure 8A) and therefore combined these time points to represent early infection . Strikingly, $91.6 \%$ of the Pgt sRNA clusters are predicted as differentially expressed among germinated spores, early infection ( 3 and $5 \mathrm{dpi}$ ) and late infection ( $7 \mathrm{dpi}$ ): 2,714 are up-regulated in germinated spores, 509 up-regulated during early infection and 3,987 up-regulated during late infection (Figure 8, Supplementary Files S5-S8). A large proportion of the up-regulated sRNAs at the late infection time point (76.1\%; 3,035 of 3,987) showed up-regulation against all the other conditions (germinated spores, 3 dpi and 5 dpi). In contrast, among the up-regulated sRNAs in germinated spores, the majority $(87.5 \% ; 2,377$ of 2,714 ) are up-regulated against the late infection sample, with only a small number (33 and 35) being upregulated compared to the 3 dpi or 5 dpi samples. Thus, the sRNAs up-regulated during late infection are highly specific to that time point, indicating the presence of early (germinated spores, $3 \mathrm{dpi}$ and $5 \mathrm{dpi}$ ) and late (7 dpi) waves of $P g t$ sRNAs during wheat infection. In contrast to $P g t$, which exhibits prominent early and late infection waves of sRNAs, only 14 of the 411 wheat sRNAs (3.4\%) are predicted to be differentially expressed. Amongst these 14 differentially expressed wheat sRNAs there is no predicted miRNA.

We assessed the length distributions and 5' nucleotide preferences of differentially expressed Pgt sRNAs (Figure 8C, D). The early wave Pgt sRNAs are predominantly $21 \mathrm{nts}$ in length (44\% and $46.2 \%$, respectively). In contrast, the largest class (40.8\%) of the late wave Pgt sRNAs are 22 nts in length. Pgt sRNAs with no detected differential expression follow a similar size distribution pattern to the early wave sRNAs, with $21 \mathrm{nt}$ sRNAs being the most prevalent class (46.4\%, Figure 8C). The majority (60-80\%) of the 20, 21 and $22 \mathrm{nt}$ sRNAs up-regulated in germinated spores, during early infection and those with no differential expression contain a 5' uracil (Figure 8D). This is also true for 20 and $21 \mathrm{nt}$ late wave sRNAs. In contrast, the $22 \mathrm{nt}$ late wave sRNAs have a strong preference for 5 ' adenines ( 70\%, Figure $8 \mathrm{D})$ ). This suggests the specific induction of a different functional class of sRNAs during these late infection stages, similar to the occurrence of $24 \mathrm{nt}$ siRNAs with a 5' adenine in plants. 
bioRxiv preprint doi: https://doi.org/10.1101/469338; this version posted July 9, 2020. The copyright holder for this preprint (which was not certified by peer review) is the author/funder, who has granted bioRxiv a license to display the preprint in perpetuity. It is made available under

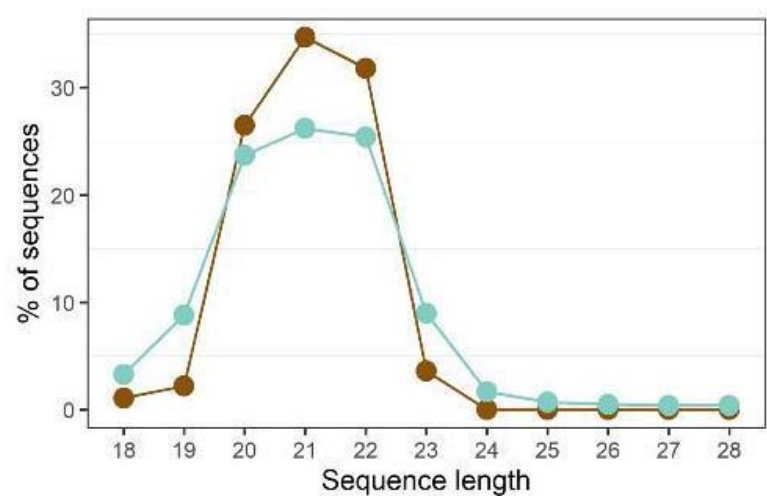

- Most abundant rust sRNA for each locus

- Read composition of rust sRNA loci

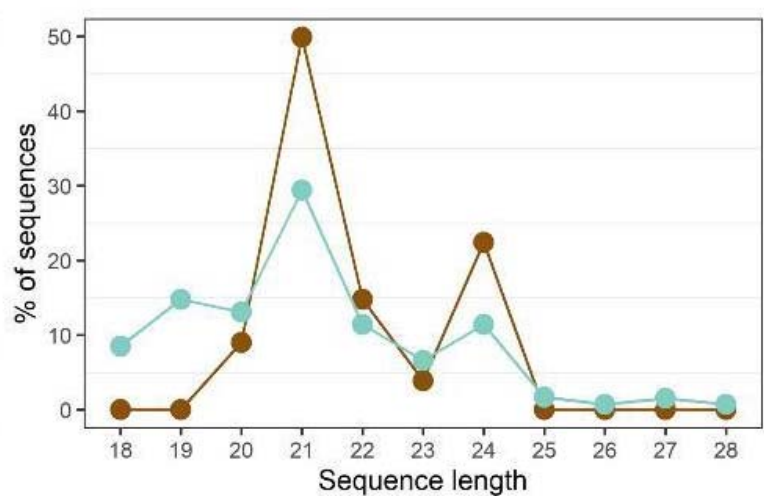

- Most abundant wheat sRNA for each locus - Read composition of wheat sRNA loci

Figure 7: Sequence length distributions of predicted sRNAs in Pgt and wheat. (A) The rust sRNAs are predominantly 20-22 nt in length, whereas the (B) wheat sRNAs show strong peaks at $21 \mathrm{nt}$ and $24 \mathrm{nt}$. Both the single most abundant RNA in each locus as well as the total reads forming the loci show the same peaks.

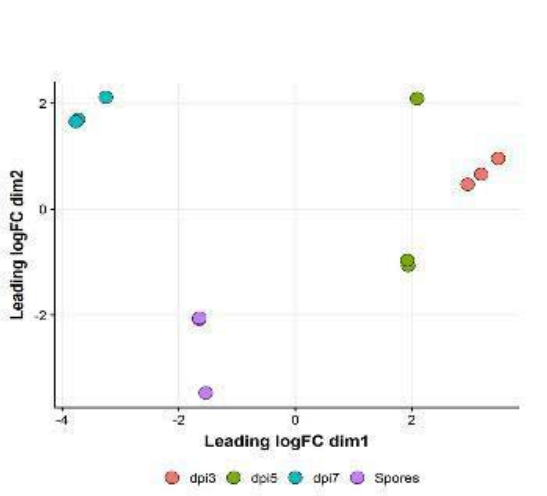

(A)

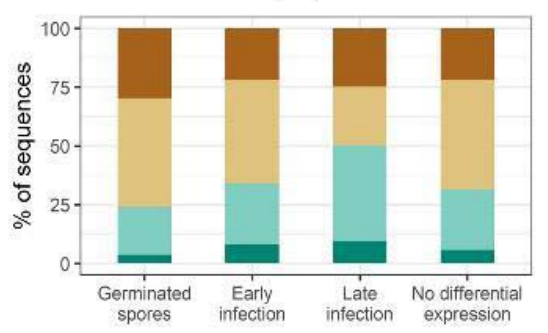

w $20 \mathrm{nt}=21 \mathrm{nt}=22 \mathrm{nt}$ Other lengths

(C)

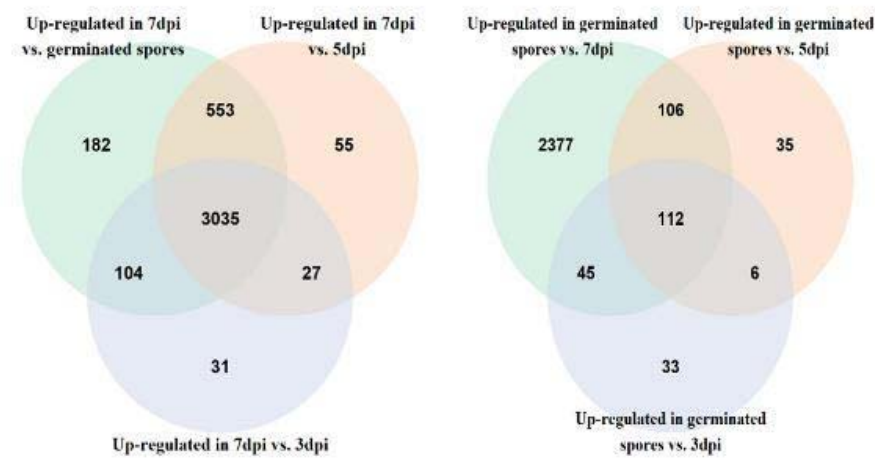

(B)

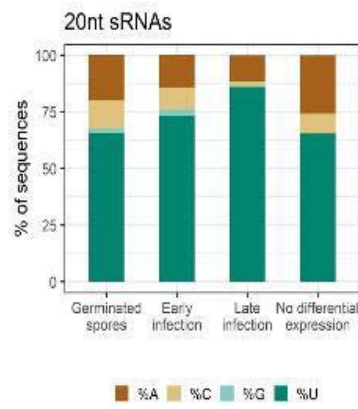

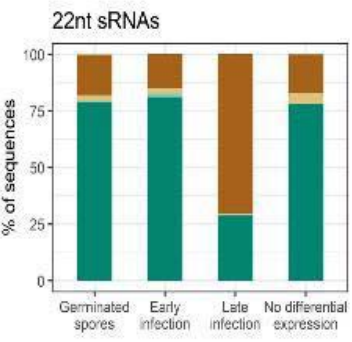

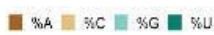

Figure 8: Pgt sRNA differentially expression analysis. (A) A multi-dimensional scaling plot using the edgeR package shows the clustering of the replicates for the different samples. The 3 dpi and 5 dpi samples show less differences in expression than the germinated spores and 7 dpi samples. (B) Venn diagrams of up-regulated Pgt sRNAs shared between the different time points: germinated spores, early infection ( 3 dpi and 5 dpi) and late infection (7 dpi). Two major classes of sRNAs occur: one that is up-regulated during late infection $(n=3,035)$ and one that is up-regulated in germinated spores compared to late infection $(n=2,377)$. (C) Sequence lengths and (D) $5^{\text {' nucleotide }}$ distribution of the Pgt sRNAs. Pgt sRNAs up-regulated during late infection differ in length distribution and $5^{\text {c }}$ 
bioRxiv preprint doi: https://doi.org/10.1101/469338; this version posted July 9, 2020. The copyright holder for this preprint (which was not certified by peer review) is the author/funder, who has granted bioRxiv a license to display the preprint in perpetuity. It is made available under aCC-BY-NC-ND 4.0 International license.

nucleotide preference to the remaining $P g t$ sRNAs. $22 \mathrm{nt} P g t$ sRNAs up-regulated during late infection strongly prefer

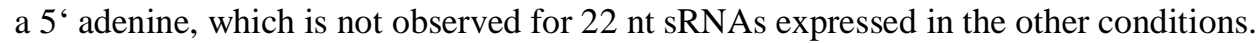

\section{Late wave Pgt sRNAs are produced from the centromeric regions, whereas the early wave sRNAs are highly conserved and derived from genes}

The late wave Pgt sRNAs also exhibit opposing genomic origins to the early wave sRNAs (Table 4). The early wave sRNAs predominantly map to annotated genes $(76.5 \%$ in germinated spores; $70.1 \%$ at 3 and 5 dpi), compared to only $16.5 \%$ of late wave sRNAs. Late wave sRNAs are largely generated from repetitive elements (86.9\%), in contrast to the early wave sRNAs $(24.2 \%$ in germinated spores and $28.7 \%$ at 3 and 5 dpi). Most of the repetitive elements associated with sRNAs belong to the class of LTR retrotransposons, particularly Gypsy elements. Strikingly, $24 \%$ of the late wave sRNAs originate from the centromeric regions, in contrast to only $1-3 \%$ of the early wave sRNAs and the sRNAs with no differential expression (Table 4).

Table 4: Genomic origins of Pgt sRNAs. The Pgt sRNAs map in similar proportions to the two haplotypes. More than half of sRNAs are conserved and have a homologous counterpart. Late wave sRNAs preferentially originate from repetitive regions and the centromeres.

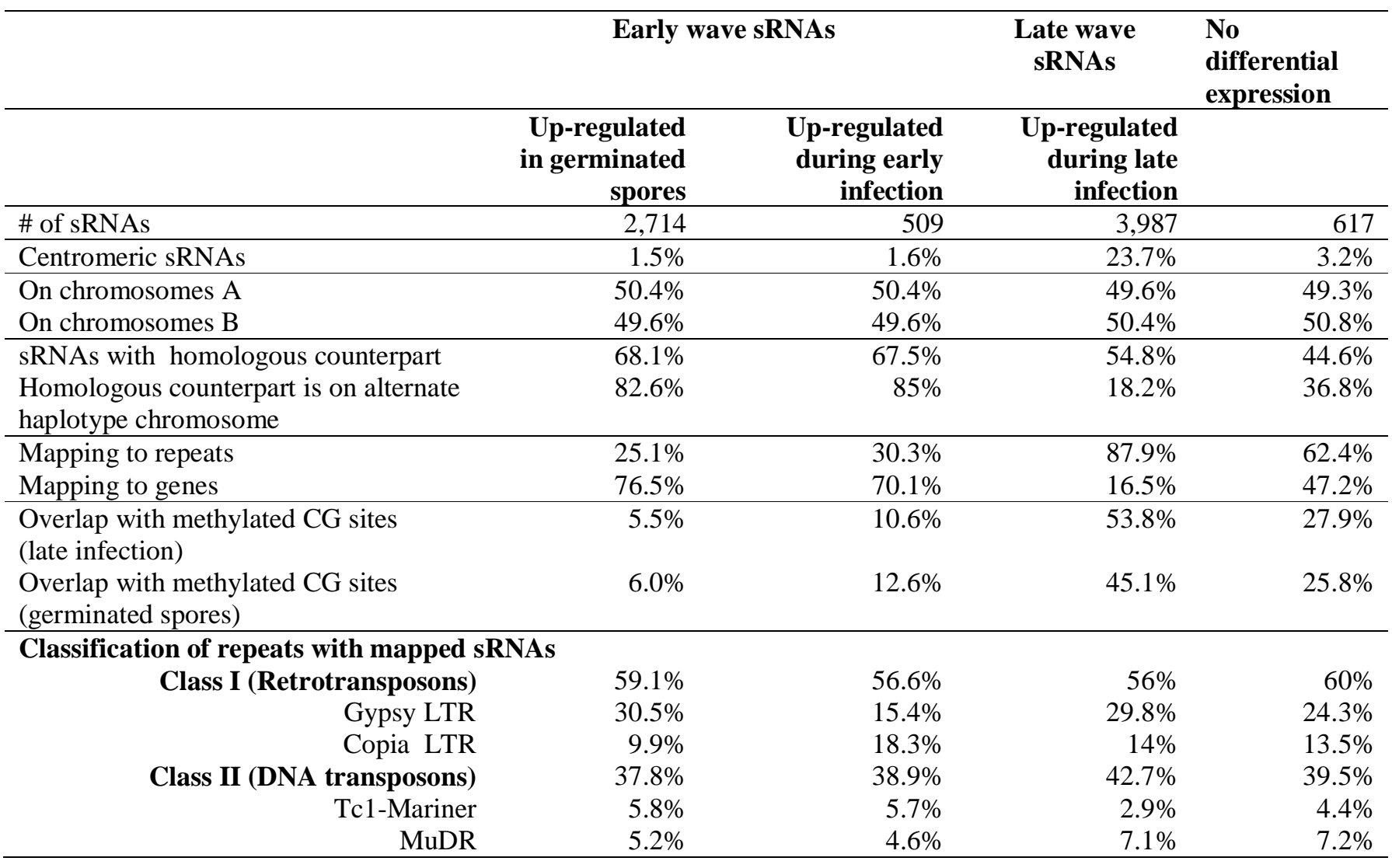

18

A gene function ontology (GO) term analysis of the 1,878 genes that are associated with Pgt sRNAs upregulated in germinated spores reveals an enrichment in proteins with ATP binding activity as well as proteins with helicase and motor activity and RNA binding (Table 5). No significant enrichment in functional annotation was observed for genes that are associated with sRNAs with no differential expression, or with sRNAs up-regulated during early or late infection.

Table 5: Pgt genes that are associated with sRNAs up-regulated in germinated spores and their functional GO term enrichment. We assessed GO term enrichments of the annotated molecular function of Pgt genes that are associated with sRNAs compared to all other Pgt genes (FDR $<0.00001$ ). The top ten categories with lowest FDR are shown. 

aCC-BY-NC-ND 4.0 International license.

\begin{tabular}{lrr}
\hline Enriched GO term category & False discovery rate (FDR) & \# of genes \\
\hline Genes that are associated with sRNAs up-regulated in germinated spores & & \\
\hline ATP binding & $1.7 \times 10^{-35}$ & 233 \\
Helicase activity & $1.5 \times 10^{-13}$ & 47 \\
Motor activity & $4.5 \times 10^{-11}$ & 25 \\
RNA binding & $6.5 \times 10^{-10}$ & 110 \\
Shikimate 3-dehydrogenase (NADP+) activity & $1.7 \times 10^{-9}$ & 8 \\
Histone methyltransferase activity (H3-K4 specific) & $4.2 \times 10^{-9}$ & 9 \\
\hline
\end{tabular}

We further investigated the locations of the Pgt sRNAs on the 18 chromosome pairs and found that similar proportions occur in each of the two haplotypes (Table 4). We then assessed if sRNAs have a homologous counterpart on the corresponding haplotype. For this we re-mapped the sequencing reads that define a sRNA locus to the remainder of the genome and assigned the sRNA locus that has the highest coverage by those mapped reads as the homologous counterpart. Over two-thirds of sRNAs up-regulated in germinated spores have a homologous counterpart (68.1\%, Table 4). Most of these homologous pairs (82.6\%) are located on the corresponding chromosome from the alternative haplotype and generally occur in syntenous positions (Supplementary Figure S5). This is consistent with the observation that most of these sRNAs map to gene sequences which are expected to occur in allelic positions in each haplotype. In contrast, only around half of the late wave sRNAs have a homologous counterpart (54.8\%), and only $18.2 \%$ of these homologous pairs are located on the corresponding chromosome (Table 4). In summary, the early wave sRNAs are conserved across the haplotypes and originate from gene models, whereas the late wave sRNAs originate from repetitive elements that are not conserved between haplotypes.

\section{During late infection, Pgt sRNAs are heavily transcribed from the centromeres and appear to direct genome-wide methylation to young TEs}

To assess sRNA transcription in the centromeres, we plotted sRNA transcription levels at late infection and in germinated spores on the chromosomes. During late infection, strong peaks of sRNA transcription are apparent in the centromeric regions, except for chromosome 11A and 11B (Figure 9). The late wave Pgt sRNAs not only originate from the centromeric regions, they are also heavily induced from the centromeres in late infection. Whilst there is also transcription of sRNAs from the centromeres in germinated spores, the centromeric peak is less apparent and the overall transcription levels in the centromeres are only about a third of that observed during late infection (average reads per million: 160 at late infection and 54 in germinated spores).

To investigate the genomic regions that might be targeted by these centromeric Pgt sRNAs, we re-mapped sRNAs without mismatches to the chromosomes and recorded all of their alignment positions. We then assessed which types of genomic regions are enriched for sRNA targeting. 30.8\% of young TEs in the centromeres and $19.3 \%$ of the young TEs outside the centromeres have a late wave sRNA mapping to them, with much lower mapping rates to young TEs observed for the other sRNA classes (Table 6). This indicates that the late wave sRNAs might be involved in the silencing of young TEs, both inside and outside the centromeres.

To address this further, we explored whether a TE that has a sRNA mapping to it $\left(\mathrm{TE}^{\mathrm{sRNA}+}\right)$ is more likely to be methylated than a TE that does not have a sRNA mapping to it $\left(\mathrm{TE}^{\mathrm{SRNA}-}\right)$. Indeed, we observed that the late wave $P g t$ sRNAs are strongly associated with methylation of TEs, particularly young TEs, both inside and outside the centromeres (Figure 10). In late infection, 33.2\% of $\mathrm{TEs}^{\mathrm{sRNA}+}$ are methylated if the overlapping sRNAs are late wave sRNAs. In contrast, only $8.3 \% \mathrm{TEs}^{\mathrm{sRNA}+}$ are methylated if the overlapping sRNAs have no differential expression. Whilst the late wave sRNAs are preferentially $22 \mathrm{nts}$ in length with a 5 ' adenine (Figure 8), only $16.7 \%$ of $\mathrm{TEs}^{\mathrm{sRNA}+}$ are methylated if they overlap with this subclass class of late wave sRNAs. This suggests that the up-regulation at late infection drives RNA-directed methylation in Pgt, and is not only restricted to the 22 nt sRNAs and a 5 ' adenine. 
Young TEs both inside and outside the centromeres appear to be particularly targeted for RNA-directed methylation by late wave sRNAs (Figure 10). Inside the centromeres, $51.3 \%$ of the TEs ${ }^{\text {sRNA+ }}$ are methylated in late infection when they overlap with late wave sRNAs. Strikingly, RNA-directed methylation is also pronounced outside the centromeres where $39.7 \%$ of the $\mathrm{TEs}^{\text {sRNA+ }}$ are methylated in late infection when they overlap with late wave infection sRNAs. Taken together, our results indicate that the late wave Pgt sRNAs originate mainly from the centromeres but seem to direct DNA methylation to loci homologous to their sequences both inside and outside the centromeres, preferentially targeting young TEs.

Table 6: Young TEs preferentially overlap with late wave sRNAs both inside and outside the centromeres.

\begin{tabular}{lrrrr}
\hline & $\begin{array}{c}\text { Young TEs in } \\
\text { centromeres }\end{array}$ & $\begin{array}{l}\text { Old TEs in } \\
\text { centromeres }\end{array}$ & $\begin{array}{l}\text { Young TEs not } \\
\text { in centromeres }\end{array}$ & $\begin{array}{l}\text { Old TEs not } \\
\text { in centromeres }\end{array}$ \\
\hline \% that are sRNA+ (germinated spores) & $3.7 \%$ & $0.7 \%$ & $3.4 \%$ & $0.5 \%$ \\
$\%$ that are sRNA+ (early infection) & $2.1 \%$ & $0.3 \%$ & $1.7 \%$ & $0.2 \%$ \\
$\%$ that are sRNA+ (late infection) & $\mathbf{3 0 . 8 \%}$ & $\mathbf{7 . 0 \%}$ & $\mathbf{1 9 . 3 \%}$ & $\mathbf{1 . 8 \%}$ \\
$\%$ that are sRNA+ (no DE) & $7.1 \%$ & $0.5 \%$ & $5.2 \%$ & $0.4 \%$ \\
\hline
\end{tabular}
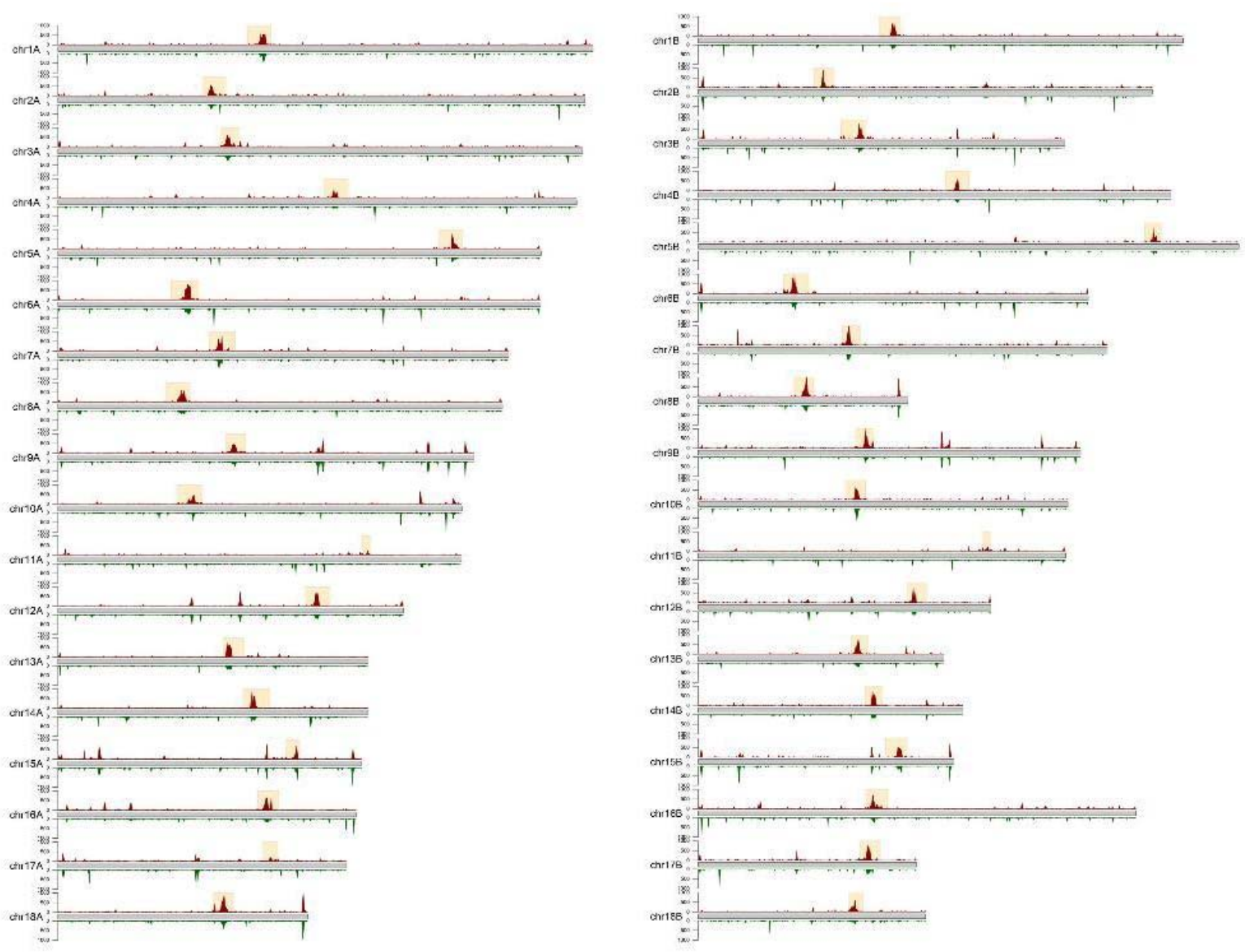

Figure 9: Transcription levels of sRNAs on the Pgt chromosomes. The transcription levels (reads per million per $10 \mathrm{~kb}$ genomic windows, < 1000 RPMs shown for clarity) of sRNAs are shown for late infection (red; above each chromosome) and for germinated spores (green; below each chromosome). Centromeric regions are indicated by yellow boxes. Higher transcription levels of sRNAs are seen from the centromeres during late infection than in germinated spores. 

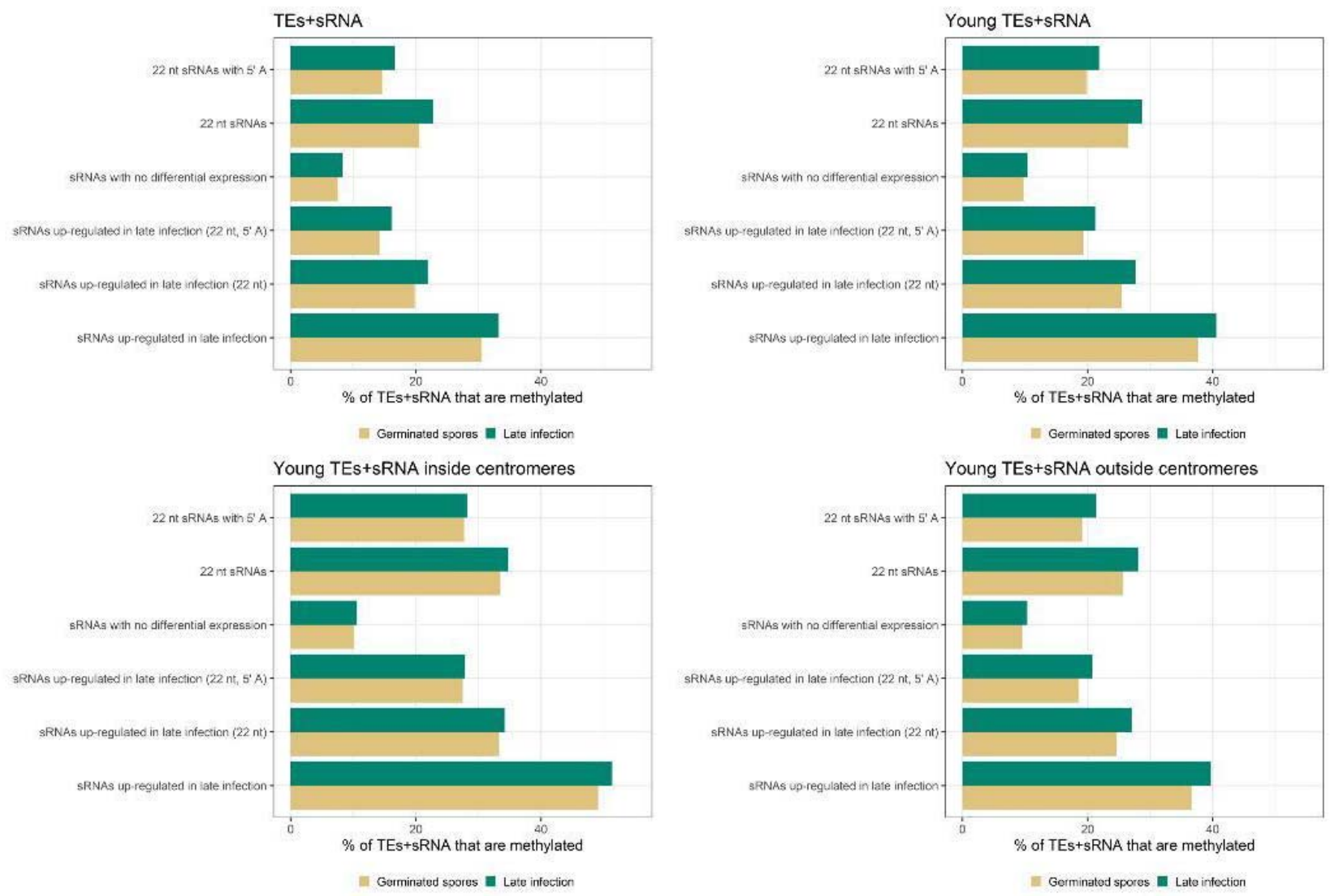

Figure 10: Percent of TEs that are methylated in germinated spores and late infection and overlap with sRNAs $\left(\right.$ TEs $\left.^{\text {SRNA+ }^{2}}\right)$. TEs both inside and outside the centromeres that overlap with late wave sRNAs $\left(\mathrm{TEs}^{\mathrm{sRNA}}\right)$ are more likely to be methylated, suggesting that $P g t$ is using sRNA-directed methylation.

\section{TE-associated CG methylation leads to silencing of nearby genes}

We investigated the effect of CG methylation and sRNA-directed CG methylation on overlapping or adjacent genes. 4,650 Pgt genes overlap with CG methylation sites in germinated spores and 4,848 genes overlap with CG methylation sites in late infection. The proteins encoded by these methylated genes are not enriched for secreted proteins or for any GO terms (data not shown). Almost all methylated genes overlap with repeats (except for 363 genes in germinated spores and 428 in late infection), suggesting that CG methylation predominantly correlates with TE silencing in Pgt.

To determine if the presence of methylated TEs affects nearby gene expression, we assessed transcription levels of genes that are close to or overlap with TEs. For both germinated spores and infected leaves, genes that have an overlapping methylated TE have significantly lower gene transcription levels than those overlapping with a non-methylated TE (Figure 11). This silencing effect is also observed for genes that are close $(<500 \mathrm{bps})$ to a methylated TE compared to genes that are close to a non-methylated TE (Figure 11A), while no difference was observed for genes > $500 \mathrm{bps}$ from methylated TEs. We then compared the expression levels of genes that overlap with TEs following whether they also overlap with the late wave sRNAs or not. Whilst genes that overlap with methylated TEs have low expression levels in general, they have significantly lower levels of expression when the TE is additionally targeted by a late wave sRNA and this holds true for both young and old TEs (Figure 11B). 

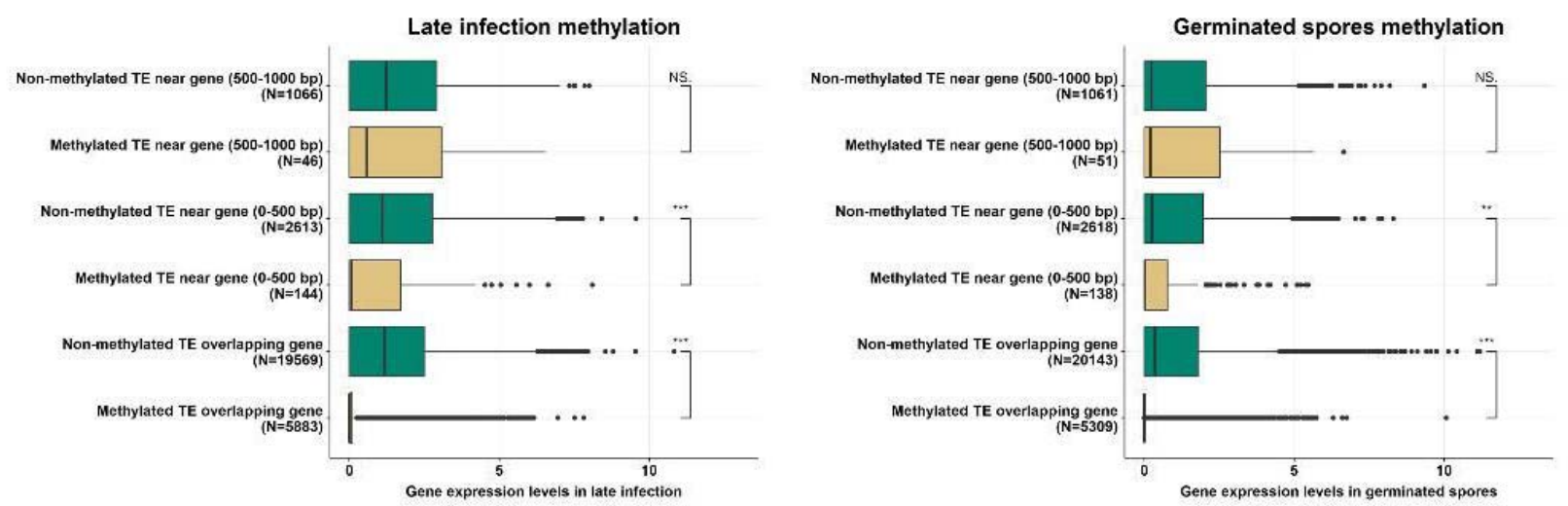

(A)
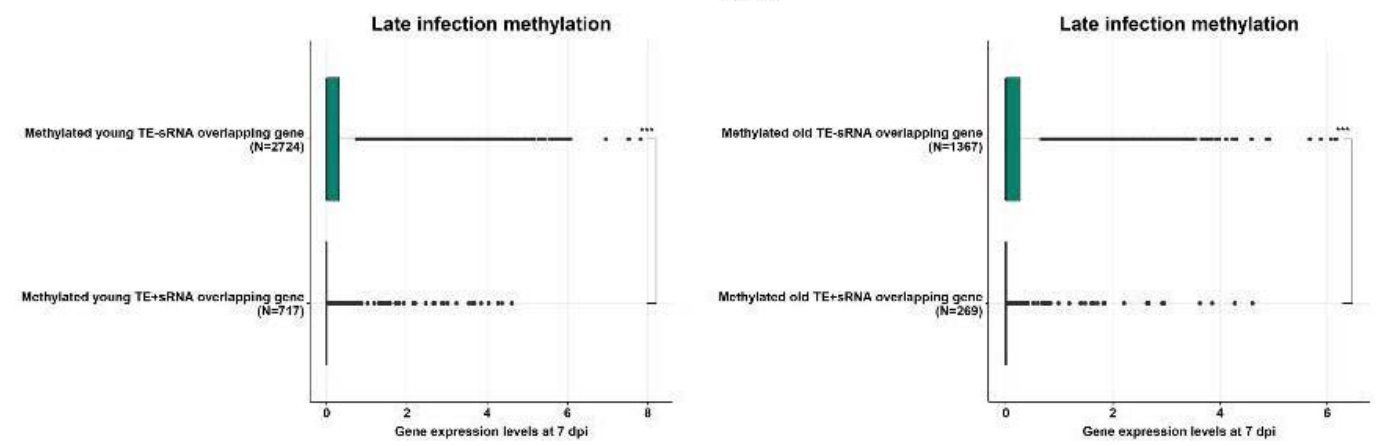

(B)

Figure 11: Expression levels of genes that have an inserted methylated repeat or that overlap with methylated repeats. (A) Expression levels (log-normalized transcripts per million) of genes are shown. A strong silencing effect is shown for genes that contain a methylated repeat, both in germinated spores and during late infection. Genes that are near a methylated repeat (up to $500 \mathrm{bps)}$ ) also show suppressed expression. (B) This gene silencing is more pronounced if a TE has a late wave sRNA mapping to it $\left(\mathrm{TEs}^{\mathrm{sRA}+}\right)$, both for young and old TEs.

\section{A subset of RNAi genes are up-regulated during late infection, supporting functional diversification}

RNAi machinery genes were previously identified in the reference genome Pgt p7a [27, 32]. We searched for the Pgt p7a RNAi genes in the gene annotation of the fully phased, chromosome-scale assembly of $P g t$ 21-0. Two argonaute genes, three dicer genes and five RdRP genes are present in the annotation of Pgt 21-0 on each haplotype (Table 8). We then searched for $5 \mathrm{mC}$ methyltransferase (5mC MTase) genes. Four classes of fungal DNA methyltransferases have been observed in fungi, but basidiomycetes predominantly have the DNMT1 $\square$ and DNMT5 genes [33]. We identified DNMT1 and DNMT5 in the Pgt 21-0 annotation by searching for the previously identified Pgt p7a genes [4] and additionally for the DNA methylase domain PFAM domain (PF00145). In line with Bewick AJ, Hofmeister BT, Powers RA, Mondo SJ, Grigoriev IV, James TY, Stajich JE and Schmitz RJ [4] we found homologs of the DNMT1 $\square$ and DNMT5 genes and confirmed the absence of the other two classes (DIM-2 and RID) and of 6mA DNA and RNA MTase genes in Pgt 21-0. The lack of 6mA DNA and RNA MTase genes in Pgt indicates that cytosine methylation is the primary DNA methylation process active in this species.

The gene expression profiles of the RNAi and 5mC MTase genes during a time course of Pgt 21-0 infecting wheat from 2-7 days post infection (dpi) [26] and in germinated spores and haustorial tissue [25] indicate two main patterns (Figure 12A): one set of RNAi genes (RdRPs 2/4/5, AGO2 and dicers 1/2) and 5mC Mtase genes that are constitutively expressed during infection, with the AGO2 genes showing particularly high expression; and another set of RNAi genes (AGO 1, dicer 3 and RdRPs 1/3) that are highly expressed only during the later stages of infection, with no or very low expression in germinated spores and during 
early infection. We did not observe differences in expression patterns of the RNAi genes between the two $P g t$ haplotypes.

A protein domain analysis further supports the functional diversification of the Pgt argonautes AGO 1 and 2. AGO 1 has an argonaute linker 1 domain and is longer in sequence, whereas AGO 2 has an argonaute linker 2 domain (Figure 12B). A phylogenetic tree constructed from the argonaute proteins of Arabidopsis thaliana and several rust fungi further supports the diversification of the rust AGOs into two classes. In Arabidopsis thaliana, AGO1 and AGO10 bind preferentially small RNAs with a 5' uracil, whereas AGO2, AGO4, AGO6, AGO7 and AGO9 prefer sRNAs with 5' adenines and AGO5 5' cytosines [1] and their diversification into these three classes is apparent from a phylogenetic tree (Figure 12C). The rust argonautes are distinct from the Arabidopsis clade and divide into two distinct families, with one copy of each present in the haploid genomes of each rust species. Taken together, the expression and sequence analyses show that Pgt RNAi machinery has functionally diversified and suggests that $P g t$ might use RNAi to regulate stage-specific infection processes, such as during the formation of new urediniospores during late infection.

Table 7: RNAi and 5mC methyltransferase genes in Pgt. For each protein, the identifiers of the allelic proteins on each haplotype are given. Homologs of the Pgt p7a PGTG_13081 and PGTG_13088 dicer proteins were not found in the gene annotation of Pgt 21-0.

\begin{tabular}{llll}
\hline $\begin{array}{l}\text { Gene } \\
\text { annotation }\end{array}$ & Identifier & Pgt 21-0 proteins & $\begin{array}{l}\text { Pgt } \\
\text { p7a identifier }\end{array}$ \\
\hline Argonautes & Argonaute 1 & PGT21_021399 (chr14A) and PGT21_022388 (chr14B) & PGTG_08429 \\
& Argonaute 2 & PGT21_001976 (chr13A) and PGT21_002123 (chr13B) & PGTG_11327 \\
\hline Dicer & Dicer 1 & PGT21_033256 (chr4A) and PGT21_033881 (chr4B) & PGTG_19535 \\
& Dicer 2 & PGT21_033021 (chr4A) and PGT21_033709 (chr4B) & PGTG_19538 \\
& Dicer 3 & PGT21_029367 (chr6A) and PGT21_028061 (chr6B) & PGTG_12289 \\
& Dicer 4 & - & PGTG_13081 \\
& Dicer 5 & - & PGTG_13088 \\
\hline RdRPs & RdRP 1 & PGT21_002642 (chr10A) and PGT21_001684 (chr10B) & PGTG_20838 \\
& RdRP 2 & PGT21_009430 (chr15A) and PGT21_009102 (chr15B) & PGTG_17766 \\
& RdRP 3 & PGT21_009651 (chr14A) and PGT21_011158 (chr14B) & PGTG_02834 \\
& RdRP 4 & PGT21_031631 (chr4A) and PGT21_032301 (chr4B) & PGTG_05092 \\
& RdRP 5 & PGT21_031875 (chr8A) and PGT21_035256 (chr16B) & PGTG_09533 \\
\hline 5mC & DNMT1 & PGT21_014413 (chr4B) and PGT21_012711 (chr4A) & PGTG_03742 \\
MTases & & & \\
& DNMT5 & PGT21_036642 (chr1A) and PGT21_037052 (chr1B) & PGTG_17071 \\
\hline
\end{tabular}




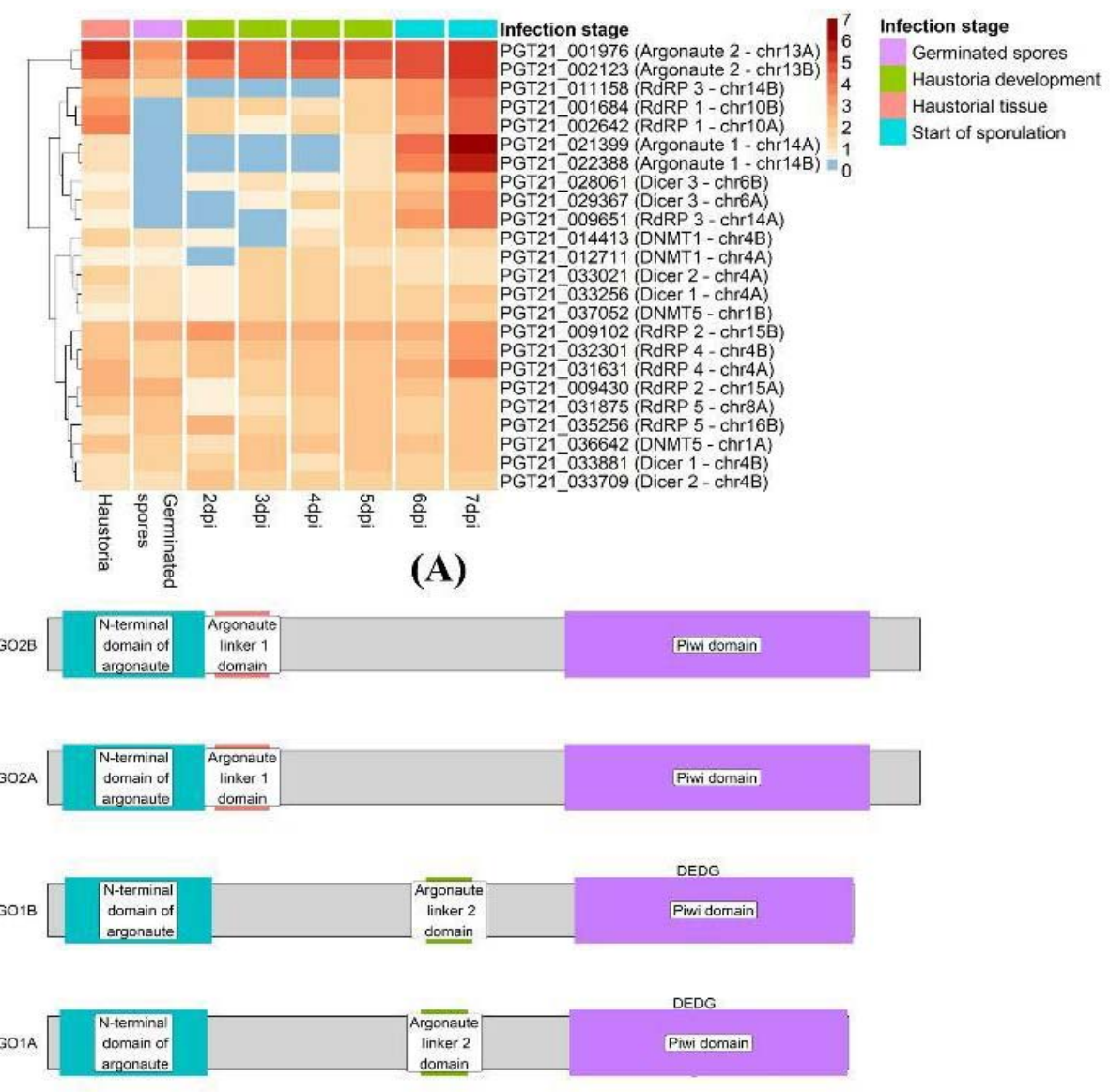

(B)

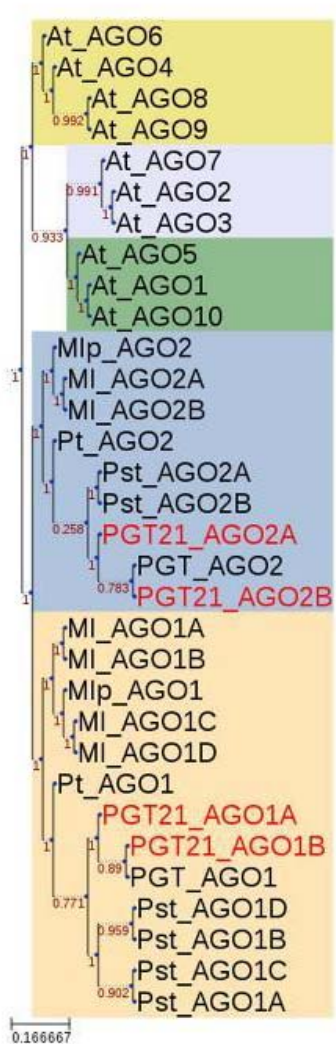

(C)

Figure 12: Pgt 21-0 RNAi and 5mC methyltransferase gene expression. (A) Hierarchical clustering of expression levels of $P g t$ RNAi genes in transcripts per million $(\log T P M$, red color intensity relates to high expression). The $P g t$ RNAi RdRPs 1/3, argonaute 1 and dicer 3 show distinct high expression at sporulation in the later stages of infection (6-7 dpi). The 5mC methyltransferases DNMT1 and DNMT5 are expressed across all conditions. (B) The Pgt argonaute proteins have diversified on the sequence level and AGO 1 and AGO 2 show differences in protein domains. (C) A phylogenetic tree of Arabidopsis argonaute proteins (At_AGO1-10) and other rust argonautes (Mlp: Melampsora larici-populina; Ml: Melampsora lini; Pt: Puccinia triticina; Pst: Puccinia striiformis f. sp. tritici; PGT: Puccinia graminis p7a) supports the diversification of cereal rust argonautes into two classes, AGO1 and AGO2.

\section{Discussion}

Epigenetic silencing mechanisms mediated by sRNAs and methylation are not well-studied in plantpathogenic fungi [10] and had thus far not been described in rust fungi. Current knowledge has been derived mainly from model species which comprise a relatively small group of fungi. Through sRNA sequencing over a time course of $P g t$-wheat infection, we uncovered that $P g t$ produces two distinct waves of sRNAs with different profiles during infection and over $90 \%$ of its sRNAs are differentially expressed. Previous studies on sRNA characterization in fungal plant pathogens mostly rely on sequencing of one time point of infection, which obscures the expression profiles of sRNAs over time. For example, a study in the stripe rust fungus Puccinia striiformis f.sp. tritici sequenced sRNAs at 4 dpi and found that the majority of the predicted 20-22 nt Pst sRNAs carry a 5 " uracil [34]. The presence of distinct sRNA profiles in mycelia and appressoria tissues was suggested in the rice blast fungal pathogen, Magnaporthe oryzae [16]. However, prominent waves of sRNA expression profiles during infection of plants had thus far not been reported.

$P g t$ sRNA expression is under tight temporal control, with $\sim 90 \%$ of $P g t$ sRNAs differentially expressed over the time course. The presence of two distinct sRNA profiles has thus far not been observed in rust fungi and supports functional diversification of the RNAi machinery, with a strong role in the infection and 
proliferation process. The early wave sRNAs are predominantly $21 \mathrm{nts}$ with a $5^{\text {' }}$ uracil derived from genes. In contrast, the late wave sRNAs are mainly $22 \mathrm{nt}$ sRNAs with a $5^{\circ}$ adenine derived from repetitive sequences. We speculate that the majority of $22 \mathrm{nt} P g t$ sRNAs are responsible for transcriptional silencing of TEs during sporulation and the majority of 20-21 nt $P g t$ sRNAs for posttranscriptional silencing of genes. This is similar to what has been reported in plants, which produces 20-22 nt miRNAs/siRNAs and $24 \mathrm{nt}$ heterochromatic sRNAs [35]. In plants, TEs are silenced mainly via 24 nt sRNAs in the RdDM pathway [1]. These 24 nt sRNAs are most abundant during seed development in plants, presumably to ensure stable inheritance of the genome.

The up-regulation of $22 \mathrm{nt} P g t$ sRNAs with enrichment for 5' adenines during late infection coincides with the up-regulation of the AGO1 gene. Similarly, the preferential accumulation of $21 \mathrm{nt} 5^{\circ}$ uracil sRNAs in germinated spores and during early infection correlates with high-level expression of $\mathrm{AGO} 2$ and relatively low expression of AGO1. This suggests that similarly to plants, the 5' nucleotide of Pgt sRNAs might have a strong effect on preferential loading into different argonautes. In Arabidopsis thaliana, AGO1 and AGO10 bind preferentially small RNAs with a 5' uracil, whereas AGO2, AGO4, AGO6, AGO7 and AGO9 prefer sRNAs with 5' adenines and AGO5 5' cytosines [1]. Our analysis suggests that Pgt AGO2 preferentially loads sRNAs with a $5^{\circ}$ uracil and AGO1 preferentially binds 22 nt sRNAs with a $5^{\circ}$ adenine, which is worthy of investigation in future experimental studies.

We discovered striking parallels between $P g t$ sRNAs and plant sRNAs, in particular compelling evidence for a sRNA-directed TE silencing pathway in $P g t$ that resembles the RdDM pathway in plants. Such a RdDM-like pathway has thus far not been reported in fungi and might suggest that Pgt uses similar strategies to plants to maintain its highly repetitive genome [36]. The overlap of the late wave Pgt sRNAs with cytosine methylation sites suggests that these sRNAs may function similarly to plant $24 \mathrm{nt}$ siRNAs to direct methylation to cause transcriptional silencing. The specific expression of one argonaute, one dicer and two RdRPs at the late stage of infection underlines their involvement in such a functionally diversified TE silencing pathway.

Furthermore, we showed that Hi-C data can be used to define centromeric regions in fungi and uncover the first centromeres in rust fungi. The Pgt centromeres are highly repetitive, hyper-methylated regions with exceptional sequence divergence, unexpectedly even between some haplotypes. Highly repetitive loci such as centromeres can generate sRNAs which in turn are required for epigenetic silencing [37]. Centromeres are essential for chromosome segregation during cell division and heterochromatin is vital to maintain the integrity of the centromeres. Eukaryotic centromere sequences are highly diverse in sequence and can differ even between closely related species [38]. In fungi, their lengths range from point centromeres ( $<400 \mathrm{bp}$ ), short regional centromeres ( $>400 \mathrm{bp},<20 \mathrm{~kb}$ ) to large regional centromeres (>20 kb) [39]. For example, the fission yeast $S$. pombe centromeres span between $35-110 \mathrm{~kb}$ and resemble those of vertebrates (central core domain of non-repetitive AT-rich DNA flanked by outer repeats), where the kinetochore is embedded in the heterochromatin of the outer repeats. In Neurospora crassa, centromeres are repetitive, AT-rich 150 to $300 \square \mathrm{kb}$ long regions [40]. The human fungal pathogen Cryptococcus harbours large regional centromeres that are rich in LTR retrotransposons [18]. The formation of silent heterochromatin in some yeasts depends on siRNAs derived from pericentromeric regions and on the RNAi machinery [12, 41]. Genes placed near centromeric chromatin are typically silenced $[42,43]$, with the strongest repression at the outer repeats [44, 45]. In the rice blast fungus Magnaporthe oryzae, centromeres span 57-kb to 109-kb transcriptionally poor regions and share highly AT-rich and heavily methylated DNA sequences [46]. Clearly, centromeres are not well-studied in plant-pathogenic fungi and had thus far not been described in rust fungi. The high activity of Pgt centromeric sRNAs in the later stages of infection might ensure that the genome is passed on stably to subsequent generations through methylation and condensation of centromeres. The TE silencing function can have a silencing effect on nearby genes, and this seems to occur in some Pgt genes that overlap with methylated TEs. In plants, insertion of TEs near genes can provide cis-elements for stress responsive or tissue-specific expression, and the expression level can be modulated by DNA methylation and/or histone modification at the TEs due to siRNA targeting. It is likely that a similar DNA methylation or histone modification mechanism exists in Pgt. 
In contrast to plants, the roles of sRNAs in epigenetic silencing pathways of fungal plant pathogens has been understudied and previous research has focused heavily on the roles of sRNAs in cross-kingdom gene silencing $[47,48]$. Several cross-kingdom RNAi interactions between fungal pathogens and plants have been uncovered. Some Botrytis cinerea sRNAs silence Arabidopsis and tomato genes involved in plant immunity and are mainly derived from LTR retrotransposons and are $21 \mathrm{nt}$ in size with a 5' uracil [49], while Arabidopsis cells secrete exosome-like extracellular vesicles to deliver sRNAs into the fungal pathogen Botrytis cinerea to silence pathogenicity genes [50]. A wheat stripe rust fungus Puccinia striiformis f. sp. tritici $20 \mathrm{nt}$ sRNA has been suggested to target the wheat defence pathogenesis-related 2 (PR2) gene [51]. The fungal pathogen Sclerotinia sclerotiorum produces mainly 22-23 nt sRNAs with a 5 ' uracil from repeatrich regions during infection [52]. Whilst $P g t$ might also use sRNAs to target host genes for silencing, we found strong support for endogenous roles of Pgt sRNAs during infection. Using the ShortStack software which uses criteria tailored to plant miRNA properties, we predicted only a handful of $P g t$ sRNAs that fulfil the criteria for miRNAs and thus might represent sRNAs involved in gene silencing. However, it is possible that Pgt produces a larger contingent of miRNA-like RNAs that follow currently unknown fungal-specific rules. Loci with some, but insufficient, evidence for miRNA biogenesis (such as strandedness) using the ShortStack software might be worth exploring as miRNA-like candidates in the future [53]. We did not perform target prediction of Pgt sRNAs due to the lack of fungal-specific targeting rules and the high false positive rate of miRNA target prediction tools [54]. In future studies, sRNA-sequencing specifically of haustorial tissues can help to elucidate if haustoria are potentially sites of sRNA transfer between the host and rust fungi [55] and then we can combine target prediction with gene expression data to reduce the number of false positive predictions.

\section{Conclusions}

The wheat stem rust disease caused by Puccinia graminis f. sp. tritici (Pgt) is one of the most devastating crop diseases and of significant global interest. Our work uncovers fundamental characteristics of the stem rust RNAi machinery, DNA methylation in rust fungi and the first characterization of centromeres in rust fungi. We found compelling evidence for an sRNA-directed DNA methylation pathway in rust fungi, similarly to the RdDM pathway in plants. Pgt induces waves of early and late infection sRNAs with differing profiles and up-regulates a subclass of RNAi genes during late infection. Future research can use this knowledge to optimize methods of host-induced gene silencing where sRNAs from the plant operate via the fungus's own RNAi machinery to silence pathogen genes important for causing disease.

\section{Methods}

\section{Hi-C data analysis and centromere identification}

Previously published Hi-C data [24] available in NCBI under BioProject PRJNA516922 was analyzed using HiC-Pro 2.11.1 [56] and contact maps were plotted with HiCExplorer's hicPlotMatrix [57] to identify centromeric regions.

Chromosomes and centromeric regions were aligned using DGenies [58] and regions of macrosynteny were extracted from the minimap2 [59] paf alignment produced by DGenies. Pairwise $k$-mer distance estimations were calculated using Mash 2.2.0 with the function mash triangle [60] and clustered as a dendogram (hclust with the method ward.D2).

\section{Gene expression analysis and repetitive element annotation}

Previously published RNA-seq data (0 dpi, 2 dpi, 3 dpi, 4 dpi, 5 dpi, 6 dpi, $7 \mathrm{dpi})$ was used for the gene expression analysis [26]. This was complemented with previously published RNA-sequencing data of Pgt 21-0 germinated spores and haustorial tissue [61]. We used Salmon 1.1.0 to align reads to the Pgt 21-0 transcripts [24] and to estimate transcript abundances in each sample (salmon index -keepDuplicates and salmon quant -validateMappings). We used tximport and DESeq2 to assess gene differential expression [62, 63]. Differentially expressed genes were annotated with the B2GO software and GO term enrichment analyses were performed with $\mathrm{B} 2 \mathrm{GO}$ and the category molecular function [64]. 
Transcription levels on the chromsomes were obtained by aligning the RNA-seq reads to the Pgt chromosomes [24] with HISAT2 2.1.0 and default parameters [65]. Bedtools 2.28.0 was used to slice the chromosomes into windows (bedtools makewindows) and the aligned reads per genomic window were counted (bedtools coverage - counts) and normalized to reads per million.

Repeat regions were annotated as described previously [66, 67] using the REPET pipeline v2.5 [29, 68, 69] for repeat annotation in combination with Repbase v21.05 [70]. For de novo identification, we predicted repeats for both haplotypes independently using TEdenovo. We combined the resulting de novo repeat libraries without removing redundancies. We annotated both haplotypes with the combined TEdenovo repeat library and two additional repeat libraries from Rebase (repbase2005_aaSeq and repbase2005_ntSeq). We generated superfamily classifications as described previously [66, 67].

\section{Methylation sequencing and analysis}

Triticum aestivum cultivar Rangcoo seeds were sown and stratified at $4{ }^{\circ} \mathrm{C}$ with no light for approximately $48 \mathrm{~h}$. To germinate, the pots were transferred to a growth cabinet set at $21^{\circ} \mathrm{C}$ with $60-70 \%$ relative humidity and a $16 \mathrm{~h}$ light cycle. Six days after sowing (seedling approximately $6 \mathrm{~cm}$ tall), plant growth inhibitor maleic hydrazide was added, $20 \mathrm{~mL}$ per pot at a concentration of $1.1 \mathrm{~g} / \mathrm{L}$. Infection of wheat seedlings was performed seven days after sowing. 300-400 mg of dormant Pgt urediniospores were heat-shock activated for approximately $3 \mathrm{~min}$ at $42-45^{\circ} \mathrm{C}$. The urediniospores were then suspended in Novec ${ }^{\mathrm{TM}} 7100$ solvent $\left(3 \mathrm{M}^{\mathrm{TM}}\right)$ and immediately used to inoculate the wheat seedlings, applying the suspension across leaves homogeneously using a flat paintbrush. Pots were then placed into a plastic container, leaves sprayed with Milli-Q water (Merck), sealed with a lid and placed in a secure transparent plastic bag. The bag was transferred to a growth cabinet set at $23^{\circ} \mathrm{C}$ with $60-70 \%$ relative humidity and a $16 \mathrm{~h}$ light cycle. After $48 \mathrm{~h}$, the plants were removed from the bag. Infected leaves (day 7) were grinded in mortar and pestle using liquid nitrogen, converted into fine powder and lysed with the lysis buffer followed by DNA binding, ethanol wash and elution. Notable changes include the addition of $6 \mathrm{mM}$ EGTA to the lysis buffer. DNA was further purified which included RNA/proteins removal, clean-up with cholorform:isoamyl alcohol (24:1), shearing with 5 passes through a 29 gauge needle and size selected with a Short Read Eliminator (SRE) XS $10 \mathrm{~kb}$ (Circulomics) according to dx.doi.org/10.17504/protocols.io.betdjei6.

To prepare material from germinated $P g t$ spores, freshly harvested spores (450-500 $\mathrm{mg}$ ) were sprinkled on top of autoclaved Milli-Q (MQ) water in a glass baking tray and were incubated at $100 \%$ humidity at $20^{\circ} \mathrm{C}$ in dark for $18 \mathrm{~h}$ before harvesting. Once germination was assessed and verified by bright field microscopy, the layer of germinated spores was collected using a glass slide and the remaining moisture was removed as much as possible with paper towel. Dried sample were snap frozen in liquid nitrogen and stored at $-80^{\circ} \mathrm{C}$ until processed for DNA extraction. High-molecular weight DNA was extracted from germinated spores following the Phenol:Chloroform method with minor modifications. Briefly, the germinated spores were grounded with liquid nitrogen into fine powdered material, suspended in lysis buffer followed by cholorform:isoamyl alcohol (24:1) steps twice, incubated in $200 \mu \mathrm{l}$ of $10 \mathrm{mM}$ Tris pH 8 and $200 \mu 1$ of TrisEDTA buffer (TE) at room temperature overnight. For secondary cleanup, DNA bound to Sera-Mag ${ }^{\mathrm{TM}}$ SpeedBead magnetic carboxylate-modified particles (GE Healthcare), washed 3 times with ethanol and eluted with $10 \mathrm{mM}$ Tris-HCl pH 8. The DNA was size selected with a Short Read Eliminator (SRE) XS 10 $\mathrm{kb}$ (Circulomics) according to dx.doi.org/10.17504/protocols.io.betdjei6.

To perform native DNA sequencing, Oxford Nanopore Technologies (ONT) portable MinION Mk1B was adopted. Native DNA sequencing libraries were constructed according to the manufacturer's protocol 1D genomic DNA by ligation (SQK-LSK109), using $3 \mu \mathrm{g}$ of DNA input. Briefly, DNA was repaired (FFPE DNA Repair Mix, New England BioLabs ${ }^{\circledR}(\mathrm{NEB})$ ), end-prepped with an adenosine overhang (Ultra II end repair / dA-tailing module, NEB), purified (AMPure XP, Beckman Coulter) and an ONT adapter was ligated each end (Quick T4 Ligation Module, NEB). Following, the library was cleaned once more and quantified using a Qubit Fluorometer (Thermo Fisher Scientific). A MinION FLO-MIN106 9.4.1 revD flow cell was 
primed, approximately $300 \mathrm{ng}$ of library was loaded and sequenced according to the manufacturer's instructions (ONT). When the majority of pores became inactive (approximately $24 \mathrm{~h}$ ), the flow cell was treated with DNase I and another $300 \mathrm{ng}$ of library was loaded, according to the ONT nuclease flush protocol. The nuclease flush protocol was performed 2-3 times, until the flow cell was expended.

\section{Bioinformatic processing and methylation calling}

Raw fast5 reads were basecalled with Guppy version 3.4.5 (ONT), using the --fast5_out option. Sequencing output and quality was inspected with the NanoPack tool NanoPlot version 1.28.2 [71]. To identify Pgt reads, we mapped all Nanopore reads from both analyzed conditions against the Pgt genome [24] using minimap2 version 2.17-r941[59] evoking the nanopore flag (-map-ont). Only reads aligning to the Pgt 21-0 reference genome were used for downstream. De novo identification of the DNA modifications $5 \mathrm{mC}$ and $6 \mathrm{~mA}$ was performed using Tombo version 1.5.1 [72]. We called $6 \mathrm{~mA}$ and $5 \mathrm{mC}$ methylation with Tombo 1.5.1 following the github instructions. We converted resulting Bigwig files into Bed6 files by calling sites methylated that had per read methylation frequency above 0.5. We called CG methylation using nanopolish 0.12.3 and pycometh v0.4.2 (https://doi.org/10.5281/zenodo.3629254). A genomic element was considered methylated if at least two methylation sites mapped to it. Di- and trinucleotide frequencies were calculated with compseq from EMBOSS 6.6.0 [73].

\section{Small RNA sequencing, read processing, filtering and alignment}

From the same infected leaf samples as the RNA-seq data [26], small RNA sequencing data was obtained. For rust infection, host plants (cv. Sonora) were grown at high density ( 25 seeds per $12 \mathrm{~cm}$ pot with compost as growth media) to the two leaf stage ( $\sim$ days) in a growth cabinet set at $18-23^{\circ} \mathrm{C}$ temperature and $16 \mathrm{~h}$ light. Spores $\left(-80^{\circ} \mathrm{C}\right.$ stock) were first thawed and heated to $42^{\circ} \mathrm{C}$ for 3 minutes, mixed with talcum powder and dusted over the plants. Pots were placed in a moist chamber for 24 hours and then transferred back to the growth cabinet. Leaf samples were harvested at specified days after inoculation, snap frozen and stored at $-80^{\circ} \mathrm{C}$ until use. $100 \mathrm{mg}$ freshly collected spores were germinated overnight in four $15 \mathrm{~cm}$ petri dishes, each containing $200 \mathrm{ml}$ sterile RO water. Germinated spores were harvested via filtering through nylon mesh $15 \mu \mathrm{m}$. Small RNAs were extracted from the germinated spores and infected leaf samples with the Purelink microRNA Isolation Kit from Invitrogen. We sequenced sRNAs (50 bp reads) from the following five conditions ( 3 replicates each) on the Illumina HiSeq: germinated spores, uninfected wheat and infected wheat at $3 \mathrm{dpi}, 5 \mathrm{dpi}$ and $7 \mathrm{dpi}$. Adapters were trimmed using cutadapt (-m18 -M28 -q30 -trim$\mathrm{n}$-discard-untrimmed) [74]. Untrimmed reads, reads shorter than $18 \mathrm{nts}$ or reads larger than 28 nts were discarded and flanking $\mathrm{N}$ bases were removed from each read [74]. FASTQC was run on the resulting reads (http://www.bioinformatics.babraham.ac.uk/projects/fastqc/).

To eliminate reads derived from non-small RNAs, we first generated a database set of potential contaminating RNA sources. Triticum aestivum and Puccinia tRNAs, rRNAs and spliceosomal RNAs were collected from the RNACentral database [75] as well as the tRNA and rRNA RFAM families RF00001, RF00002, RF00005, RF01852, RF01960 and RF02543 [76], snoRNAs from dbsnOPY, 5S and 23S ribosomal RNAs from the European Nucleotide Archive (ENA) and the tRNA/rRNA file from the sRNA workbench [77]. This set of potential contaminant sequences was de-duplicated using bbmap and its tool dedupe.sh (sourceforge.net/projects/bbmap/). Reads that mapped to this set were removed using bowtie 1.1.2 [78]. To assess read length distributions across the different samples, clean small RNA reads were mapped to the wheat genome IWGSC RefSeq v1.0 [79] and PGT 21-0 genome [24] using bowtie 1.1.2 (alignment settings: no mismatches allowed -v0; report all alignments: -a -best -strata). To annotate highconfidence Pgt and wheat sRNAs from the sequencing data, we used the ShortStack software [80]. ShortStack predicts and quantifies sRNA-producing loci in a genome based on clusters of sRNA reads and miRNA-producing loci according to a series of tests, such as strandedness of the locus and the predicted precursor secondary structure.

\section{Pgt sRNA prediction, differential expression analysis and allelic sRNA prediction}


To annotate and quantify high-confidence $P g t$ and wheat small RNAs from the sequencing data, we used the ShortStack 3.8.5 software [80] on the clean sRNA reads (--bowtie_m all). We further filtered the predicted sRNA clusters to include only those where $>=80 \%$ of reads are within $20-24$ nts of length (recommended procedure in ShortStack to avoid degradation products) and where the cluster has $>=5$ reads per million. The ShortStack software outputs sRNA cluster properties such as the most abundant sRNA (termed sRNA candidate) in the cluster, strandedness of the locus, miRNA annotation and phasing [80]. Strandedness of sRNA loci is determined by forcing the bowtie aligner to select one strand or the other with a probability that is proportional to the number of best sites on the strand. Stranded loci are typical of miRNA production in plants and are a requirement for annotation of a locus as a miRNA by ShortStack. We used the read counts returned by ShortStack for all predicted sRNA clusters and used edgeR [81] to assess which are differentially expressed at any of the infection stages versus germinated spores (FDR $<0.05$, fold change $>$ 2).

To assess if sRNAs have a homologous counterpart, we re-mapped the sequencing reads that define a sRNA locus to the remainder of the genome using bowtie 1.1.2 (alignment settings: two mismatches allowed -v2; report all alignments: -a -best -strata). If more than $25 \%$ of bases in a sRNA locus are covered by those mapped reads (using bedtools coverage version 2.28.0), it is marked as a candidate homolog. The sRNA locus with the highest coverage amongst the candidate homologs is returned as the predicted allelic counterpart. Circos 0.69.5 [82] was used to plot the links between homologous sRNAs across the chromosomes.

To assess the relationships of sRNAs and TEs, we re-mapped sRNAs to the genome using bowtie 1.1.2 (alignment settings: no mismatches allowed -v0; report all alignments: -a -best -strata). We reported repeats that overlap with those mapped sRNAs using bedtools intersect [83]. We then retrieved the genes that overlap with repeats using bedtools closest.

All plots were produced using Ggplot2 (Wickham, 2009) and statistical significance was assessed with $t \square$ tests using the ggsignif package (https://cran.r-project.org/web/packages/ggsignif/index.html). Significance thresholds according to $t \square$ test are: NS, not significant; *, $<0.05$; **, $<0.01$; ***, $<0.001$.

\section{Phylogenetic tree of RNAi genes}

Argonaute protein sequences were aligned with MUSCLE 3.8.31 [84] and default parameters. FastTree 2.1.9 [85] was used to construct a phylogenetic tree from the protein sequence alignment (-pseudo -spr 4 -mlacc 2 -slownni). ETE 3.1.1 was used to draw the phylogenetic tree [86].

\section{Declarations}

\section{Ethics approval and consent to participate}

Not applicable

\section{Consent for publication}

Not applicable

\section{Availability of data and materials}

All scripts as well as code for generating the figures of this paper are available at https://github.com/JanaSperschneider/Publications_Code/tree/master/2019_12_StemRust_smRNA_Paper.

Sequence data for the Pgt infection RNAseq is available at the National Center for Biotechnology Information Sequencing Read Archive under Bioproject PRJNA415866. Sequence data for the Pgt small RNAseq is available at CSIRO Data Access Portal under https://doi.org/10.25919/5bd93643b1e41. Hi-C data is available in NCBI under BioProject PRJNA516922. Methylation data is available at SRA under SUB7741177.

\section{Competing interests}

The authors declare that they have no competing interests. 
bioRxiv preprint doi: https://doi.org/10.1101/469338; this version posted July 9, 2020. The copyright holder for this preprint (which was not certified by peer review) is the author/funder, who has granted bioRxiv a license to display the preprint in perpetuity. It is made available under aCC-BY-NC-ND 4.0 International license.

13

\section{Funding}

JS is supported by an Australian Research Council Discovery Early Career Researcher Award 2019 (DE190100066). BS is supported by an ARC Future Fellowship (FT180100024). We acknowledge funding support from the 2Blades Foundation.

\section{Authors' contributions}

JS, JMT and PND conceived the study and designed experiments. JS analysed and interpreted all data sets and wrote the manuscript. AWJ, JN and BS performed methylation sequencing experiments and analysed methylation data. BX performed small RNA sequencing experiments. SJ, NMU and RM performed experiments and all authors contributed to data analysis and manuscript writing. All authors read and approved the final manuscript.

\section{Acknowledgements}

We thank Xiaodi Xia for excellent technical assistance.

\section{Supplementary Figures}
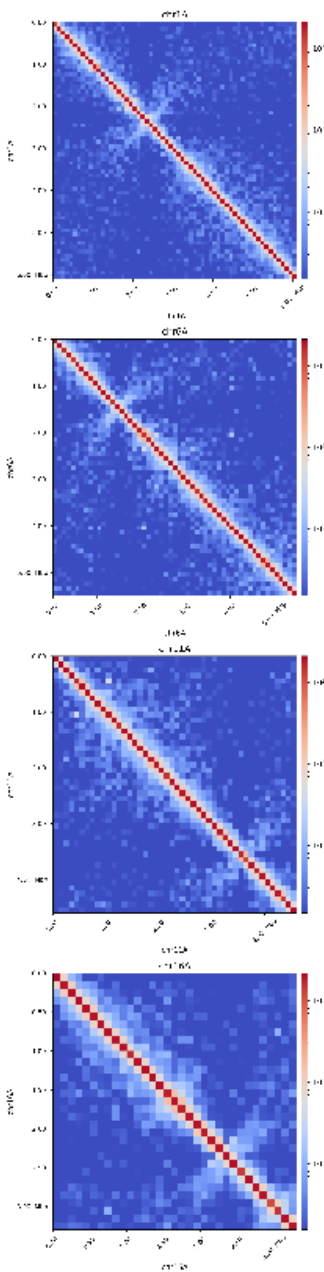
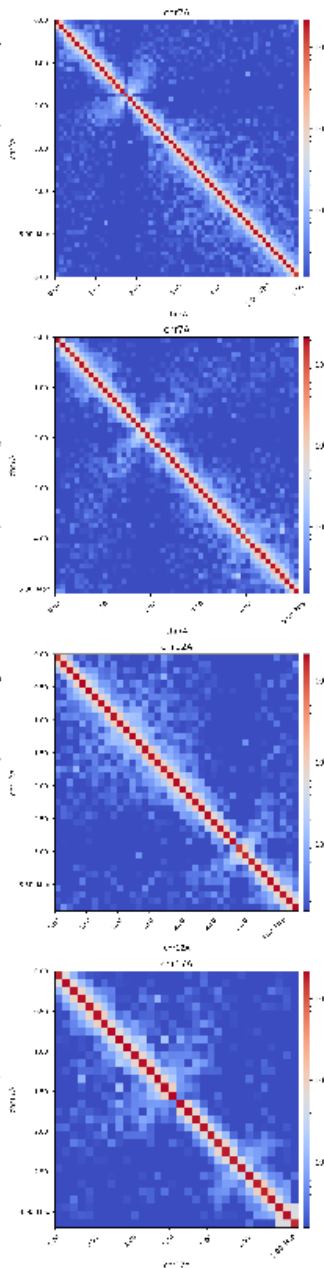

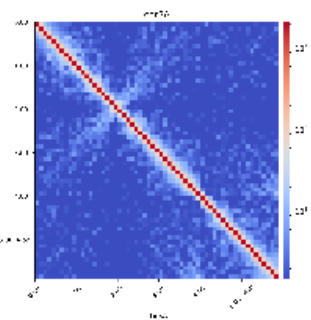

cas

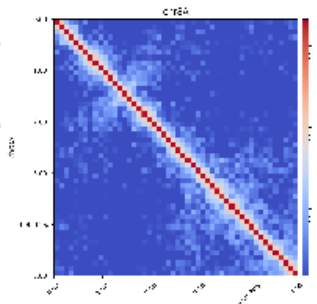

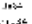
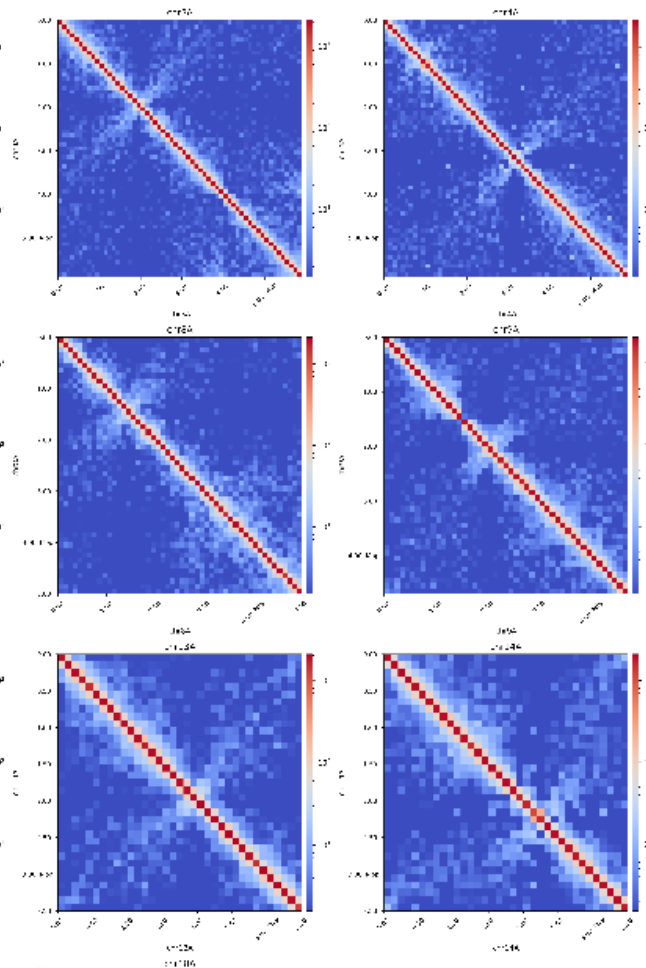

r:
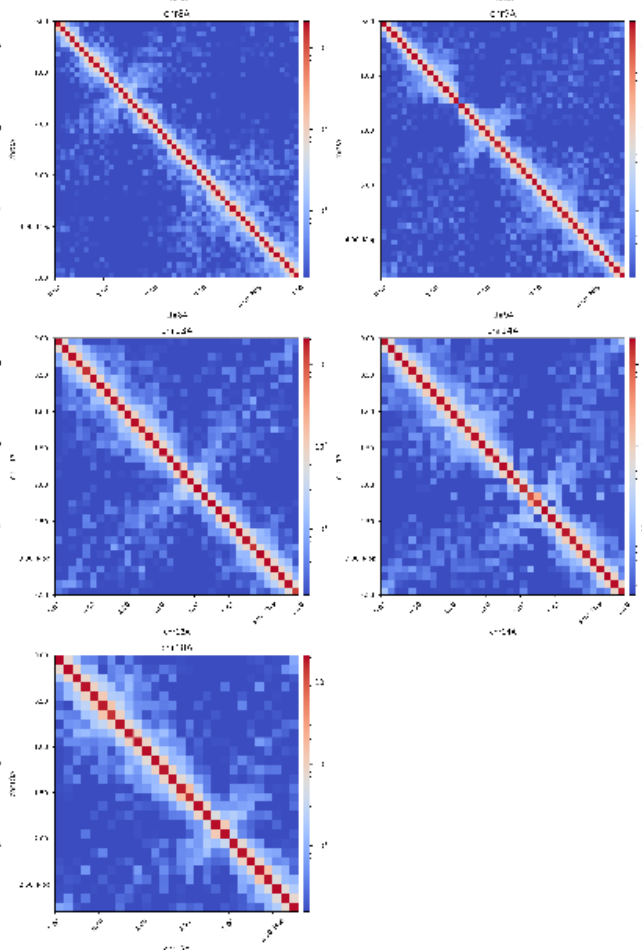
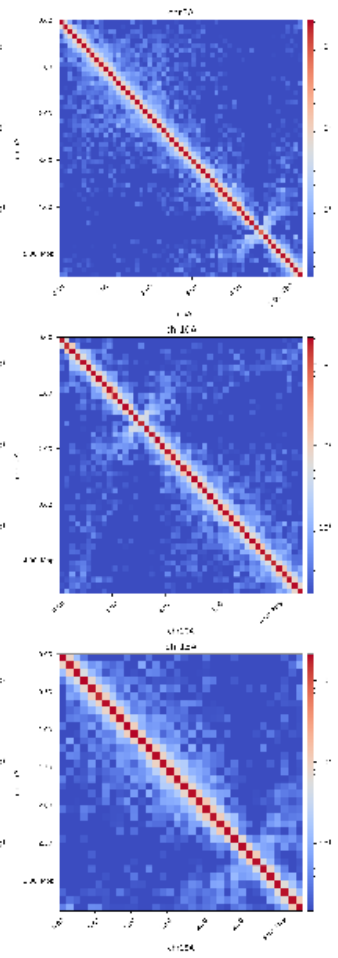

Figure S1: Hi-C contact maps for the 18 chromosomes of haplotype A show the presence of centromeres in each chromosome. 
bioRxiv preprint doi: https://doi.org/10.1101/469338; this version posted July 9, 2020. The copyright holder for this preprint (which was not certified by peer review) is the author/funder, who has granted bioRxiv a license to display the preprint in perpetuity. It is made available under
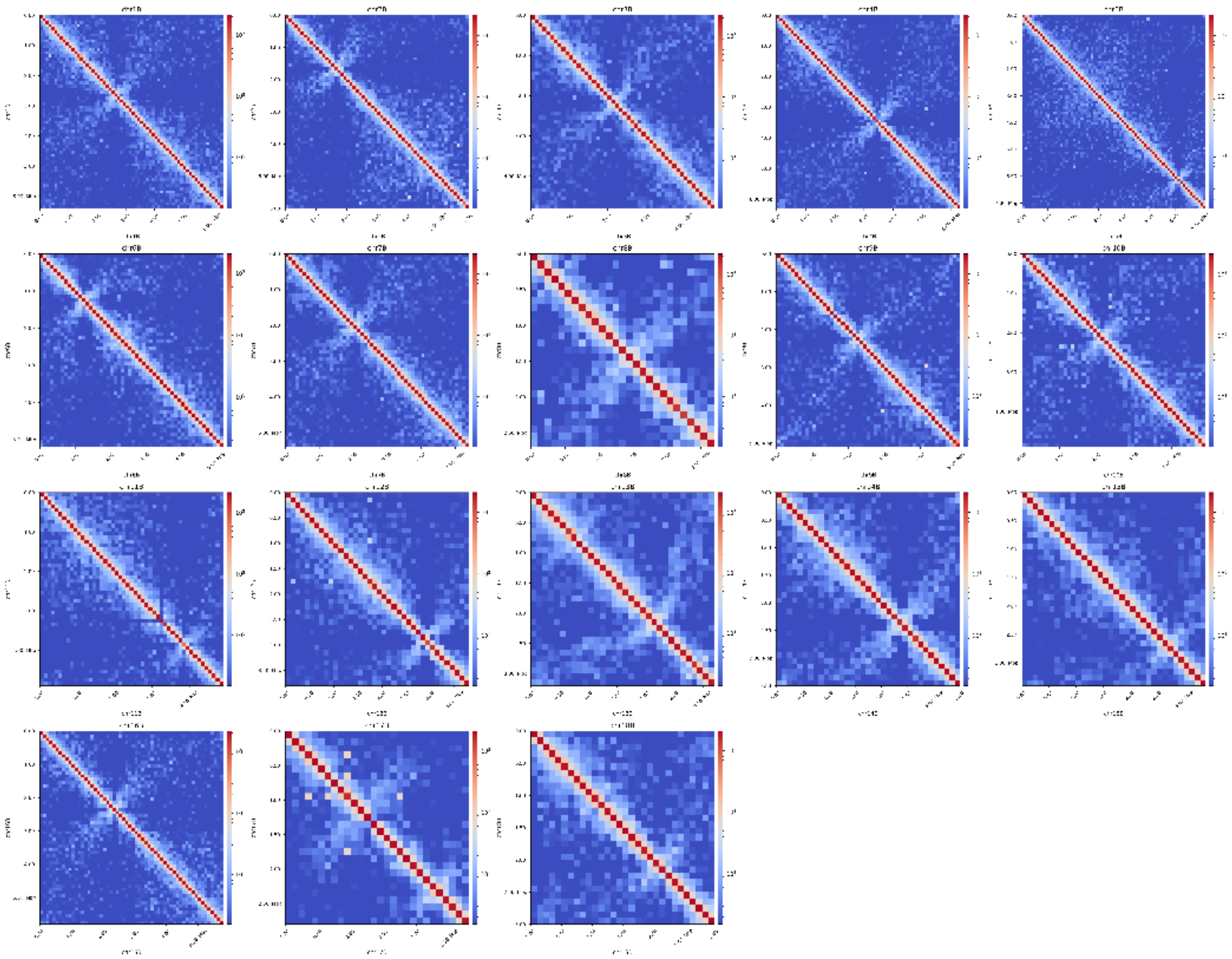

Figure S2: Hi-C contact maps for the 18 chromosomes of haplotype B show the presence of centromeres in each chromosome.

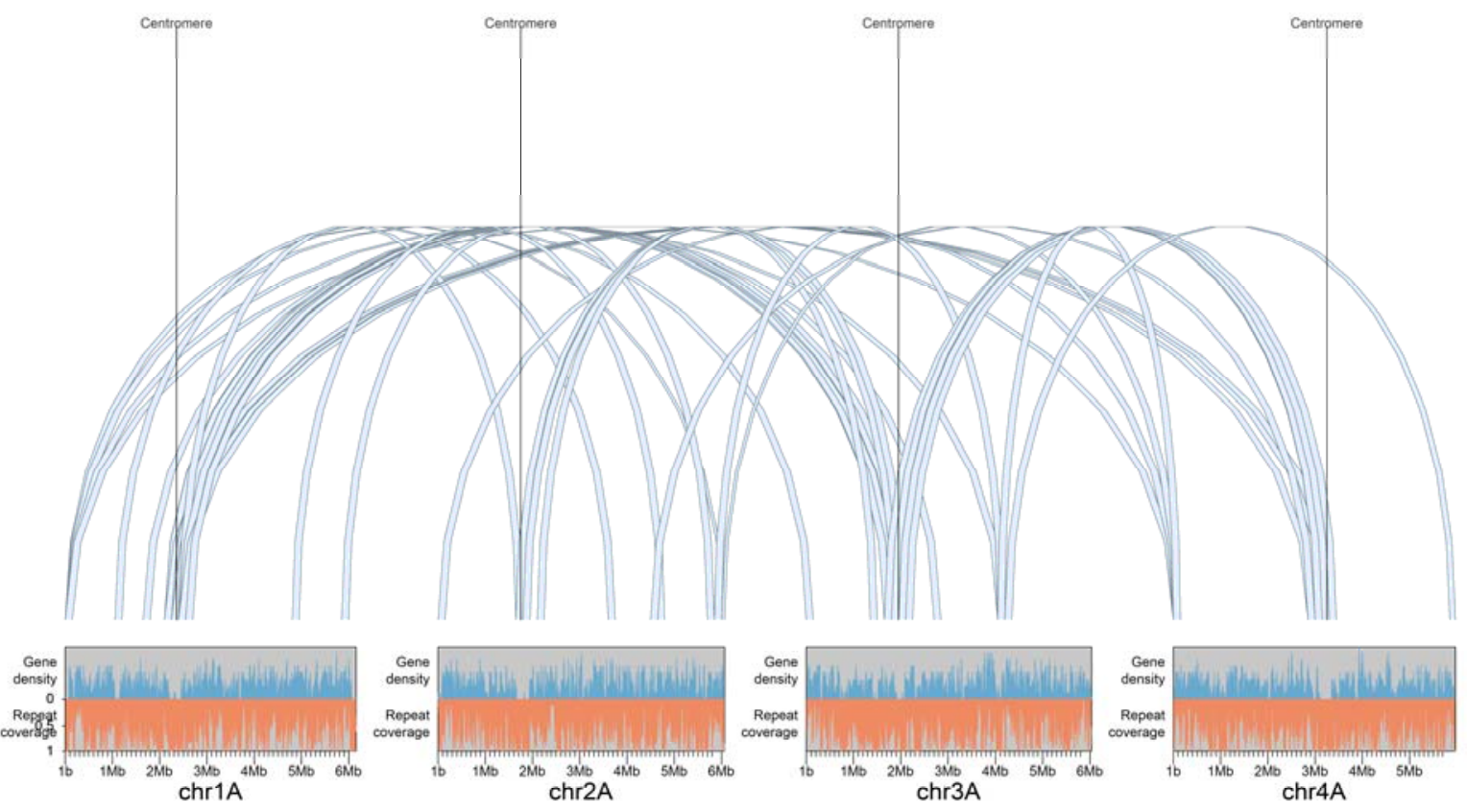

Figure S3: $150 \mathrm{kbp}$ bins with interaction frequency $>5$ in the $\mathrm{Hi}-\mathrm{C}$ interaction matrix are shown between chromosomes $1 \mathrm{~A}, 2 \mathrm{~A}, 3 \mathrm{~A}$ and $4 \mathrm{~A}$. The putative centromeric regions share strong connections with each 
bioRxiv preprint doi: https://doi org/10.1101/469338; this version posted July 9,2020 . The copyright holder for this preprint (which was not certified by peer review) is the author/funder, who has granted bioRxiv a license to display the preprint in perpetuity. It is made available under aCC-BY-NC-ND 4.0 International license.

other. Densities of expressed genes and coverage of repetitive elements are shown with window size $10 \mathrm{kbp}$. The centromeric regions are gene-poor regions with high repetitive element coverage.

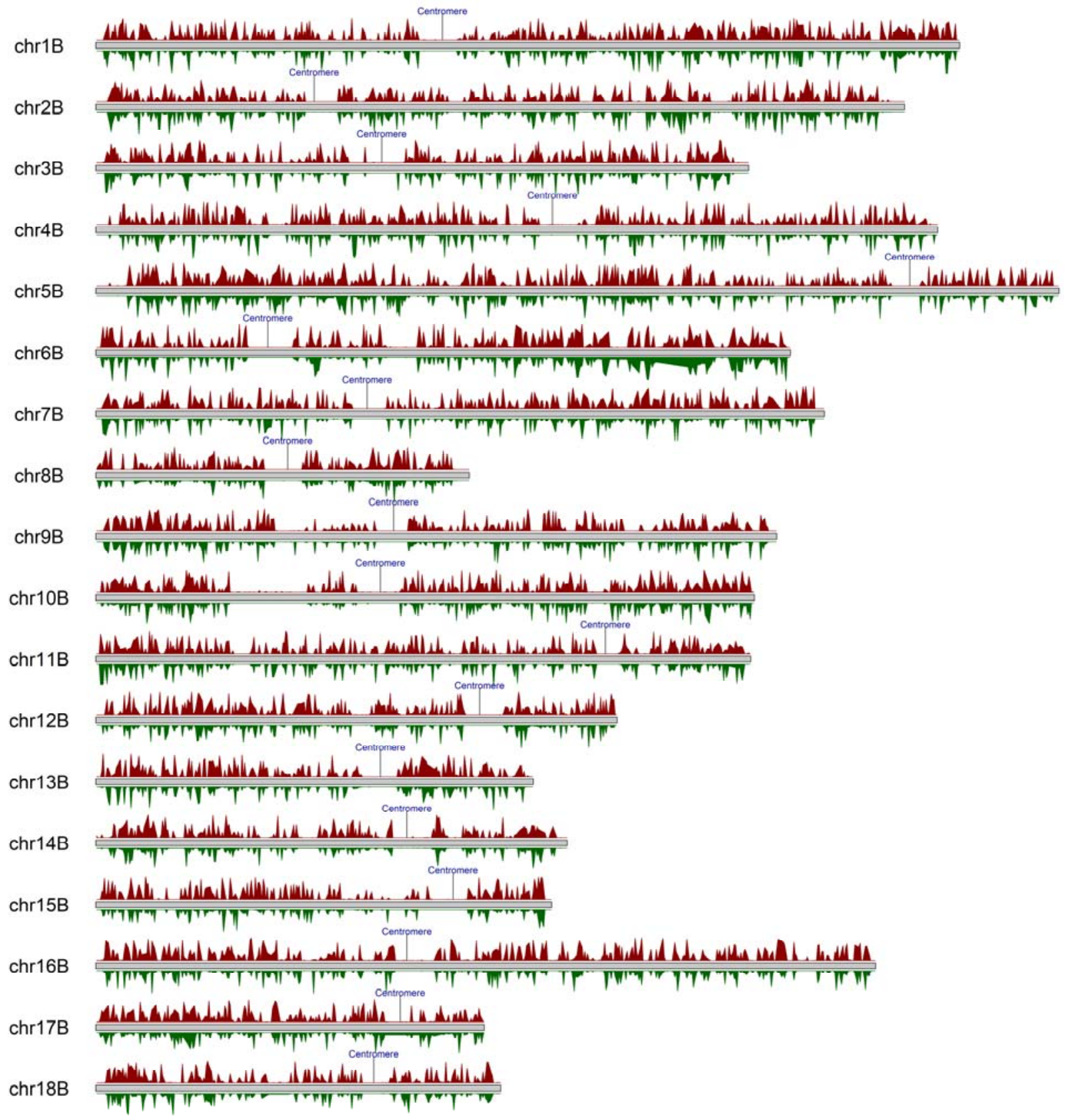

Figure S4: The positions of the centromeres in haplotype B as indicated by the Hi-C contact map are in transcriptionally silent genomic regions. Reads per million (RPM) for the late infection (7 dpi) and germinated spores RNAseq samples are shown in red and green, respectively (10 kb windows, RPM from 0100 are shown for clarity). 


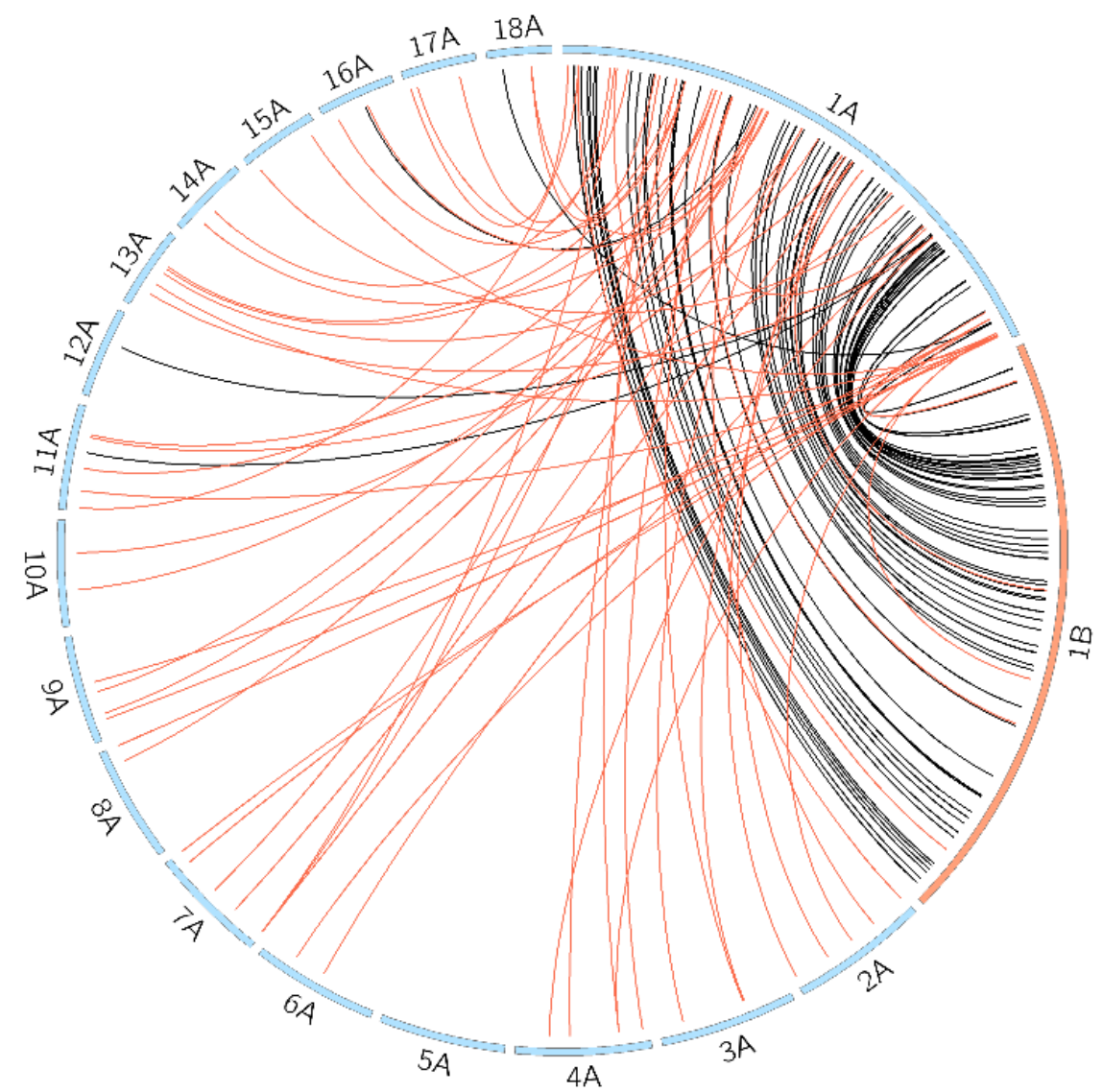

Figure S5: $P g t$ allelic sRNA pairs and their genomic localization for chromosome 1A. Pgt sRNAs that are up-regulated in germinated spores (late infection) and their homologous counterparts are shown with black (red) links. sRNAs that are up-regulated in germinated spores appear to be in syntenic on the two haplotype chromosomes $1 \mathrm{~A}$ and $1 \mathrm{~B}$ (shown at twice their size, other chromosomes shown at 0.2 their size). In contrast, sRNAs that are up-regulated during late infection on chromosome 1A have homologous counterparts on all other chromosomes except $5 \mathrm{~A}$ and $12 \mathrm{~A}$.

\section{Supplementary Files}

Supplementary File S1: FASTA file of predicted $P g t$ siRNAs.

Supplementary File S2: FASTA file of predicted Pgt miRNAs.

Supplementary File S3: FASTA file of predicted wheat siRNAs.

Supplementary File S4: FASTA file of predicted wheat miRNAs.

Supplementary File S5: FASTA file of Pgt sRNAs predicted to be up-regulated in germinated spores.

Supplementary File S6: FASTA file of $P g t$ sRNAs predicted to be up-regulated in $3 \mathrm{dpi}$ and/or $5 \mathrm{dpi}$.

Supplementary File S7: FASTA file of Pgt sRNAs predicted to be up-regulated in 7 dpi.

Supplementary File S8: FASTA file of Pgt sRNAs predicted to have no differential expression.

\section{$\underline{\text { References }}$}


1. Borges F, Martienssen RA: The expanding world of small RNAs in plants. Nat Rev Mol Cell Biol 2015, 16:727741.

2. Czech B, Hannon GJ: Small RNA sorting: matchmaking for Argonautes. Nat Rev Genet 2011, 12:19-31.

3. Martienssen R, Moazed D: RNAi and Heterochromatin Assembly. Cold Spring Harbor Perspectives in Biology 2015, 7

4. Bewick AJ, Hofmeister BT, Powers RA, Mondo SJ, Grigoriev IV, James TY, Stajich JE, Schmitz RJ: Diversity of cytosine methylation across the fungal tree of life. Nat Ecol Evol 2019, 3:479-490.

5. Freitag M, Selker EU: Controlling DNA methylation: many roads to one modification. Curr Opin Genet Dev 2005, 15:191-199.

6. Matzke MA, Mosher RA: RNA-directed DNA methylation: an epigenetic pathway of increasing complexity. Nat Rev Genet 2014, 15:394-408.

7. Xie M, Yu B: siRNA-directed DNA Methylation in Plants. Curr Genomics 2015, 16:23-31.

8. Lister R, O'Malley RC, Tonti-Filippini J, Gregory BD, Berry CC, Millar AH, Ecker JR: Highly integrated singlebase resolution maps of the epigenome in Arabidopsis. Cell 2008, 133:523-536.

9. Cuerda-Gil D, Slotkin RK: Non-canonical RNA-directed DNA methylation. Nat Plants 2016, 2:16163.

10. Dubey A, Jeon J: Epigenetic regulation of development and pathogenesis in fungal plant pathogens. Molecular Plant Pathology 2017, 18:887-898.

11. Chang SS, Zhang Z, Liu Y: RNA interference pathways in fungi: mechanisms and functions. Annu Rev Microbiol 2012, 66:305-323.

12. Volpe TA, Kidner C, Hall IM, Teng G, Grewal SI, Martienssen RA: Regulation of heterochromatic silencing and histone H3 lysine-9 methylation by RNAi. Science 2002, 297:1833-1837.

13. Volpe T, Schramke V, Hamilton GL, White SA, Teng G, Martienssen RA, Allshire RC: RNA interference is required for normal centromere function in fission yeast. Chromosome Research 2003, 11:137-146.

14. Aramayo R, Selker EU: Neurospora crassa, a model system for epigenetics research. Cold Spring Harb Perspect Biol 2013, 5:a017921.

15. Romano N, Macino G: Quelling: transient inactivation of gene expression in Neurospora crassa by transformation with homologous sequences. Mol Microbiol 1992, 6:3343-3353.

16. Nunes CC, Gowda M, Sailsbery J, Xue M, Chen F, Brown DE, Oh Y, Mitchell TK, Dean RA: Diverse and tissueenriched small RNAs in the plant pathogenic fungus, Magnaporthe oryzae. BMC Genomics 2011, 12:288.

17. Borgognone A, Castanera R, Morselli M, Lopez-Varas L, Rubbi L, Pisabarro AG, Pellegrini M, Ramirez L: Transposon-associated epigenetic silencing during Pleurotus ostreatus life cycle. DNA Res 2018, 25:451464.

18. Yadav V, Sun S, Billmyre RB, Thimmappa BC, Shea T, Lintner R, Bakkeren G, Cuomo CA, Heitman J, Sanyal K: RNAi is a critical determinant of centromere evolution in closely related fungi. Proc Natl Acad Sci U $S A$ 2018, 115:3108-3113.

19. Dubin HJB, J.P.: Combating stem and leaf rust of wheat: Historical perspective, impacts, and lessons learned. International Food Policy Research Institute 2009.

20. Figueroa M, Upadhyaya NM, Sperschneider J, Park RF, Szabo U, Steffenson B, Ellis JG, Dodds PN: Changing the Game: Using Integrative Genomics to Probe Virulence Mechanisms of the Stem Rust Pathogen Puccinia graminis f. sp. tritici. Front Plant Sci 2016, 7:205.

21. Leonard KJ, Szabo L: Stem rust of small grains and grasses caused by Puccinia graminis. Mol Plant Pathol 2005, 6:99-111.

22. Garnica DP, Nemri A, Upadhyaya NM, Rathjen JP, Dodds PN: The ins and outs of rust haustoria. PLoS Pathog 2014, 10:e1004329.

23. Hacquard S, Petre B, Frey P, Hecker A, Rouhier N, Duplessis S: The poplar-poplar rust interaction: insights from genomics and transcriptomics. J Pathog 2011, 2011:716041.

24. Li F, Upadhyaya NM, Sperschneider J, Matny O, Nguyen-Phuc H, Mago R, Raley C, Miller ME, Silverstein KAT, Henningsen $\mathrm{E}$, et al: Emergence of the Ug99 lineage of the wheat stem rust pathogen through somatic hybridisation. bioRxiv 2019:692640.

25. Upadhyaya NM, Garnica DP, Karaoglu H, Sperschneider J, Nemri A, Xu B, Mago R, Cuomo CA, Rathjen JP, Park RF, et al: Comparative genomics of Australian isolates of the wheat stem rust pathogen Puccinia graminis f. sp. tritici reveals extensive polymorphism in candidate effector genes. Front Plant Sci 2015, 5:759. 

aCC-BY-NC-ND 4.0 International license.

26. Chen J, Upadhyaya NM, Ortiz D, Sperschneider J, Li F, Bouton C, Breen S, Dong C, Xu B, Zhang X, et al: Loss of AvrSr50 by somatic exchange in stem rust leads to virulence for Sr50 resistance in wheat. Science 2017, 358:1607-1610.

27. Duplessis S, Cuomo CA, Lin YC, Aerts A, Tisserant E, Veneault-Fourrey C, Joly DL, Hacquard S, Amselem J, Cantarel $\mathrm{BL}$, et al: Obligate biotrophy features unraveled by the genomic analysis of rust fungi. Proc Natl Acad Sci U S A 2011, 108:9166-9171.

28. Varoquaux N, Liachko I, Ay F, Burton JN, Shendure J, Dunham MJ, Vert JP, Noble WS: Accurate identification of centromere locations in yeast genomes using Hi-C. Nucleic Acids Res 2015, 43:5331-5339.

29. Maumus F, Quesneville $\mathrm{H}$ : Ancestral repeats have shaped epigenome and genome composition for millions of years in Arabidopsis thaliana. Nature Communications 2014, 5.

30. Simpson JT, Workman RE, Zuzarte PC, David M, Dursi L, Timp W: Detecting DNA cytosine methylation using nanopore sequencing. Nature Methods 2017, 14:407-+.

31. Geng S, Kong X, Song G, Jia M, Guan J, Wang F, Qin Z, Wu L, Lan X, Li A, Mao L: DNA methylation dynamics during the interaction of wheat progenitor Aegilops tauschii with the obligate biotrophic fungus Blumeria graminis f. sp. tritici. New Phytol 2019, 221:1023-1035.

32. Choi J, Kim KT, Jeon J, Wu J, Song $\mathrm{H}$, Asiegbu FO, Lee YH: funRNA: a fungi-centered genomics platform for genes encoding key components of RNAi. BMC Genomics 2014, 15 Suppl 9:S14.

33. Schmitz RJ, Lewis ZA, Goll MG: DNA Methylation: Shared and Divergent Features across Eukaryotes. Trends Genet 2019, 35:818-827.

34. Mueth NA, Ramachandran SR, Hulbert SH: Small RNAs from the wheat stripe rust fungus (Puccinia striiformis f.sp. tritici). BMC Genomics 2015, 16:718.

35. Kamthan A, Chaudhuri A, Kamthan M, Datta A: Small RNAs in plants: recent development and application for crop improvement. Front Plant Sci 2015, 6:208

36. Raffaele $S$, Kamoun S: Genome evolution in filamentous plant pathogens: why bigger can be better. Nat Rev Microbiol 2012, 10:417-430.

37. van Wolfswinkel JC, Ketting RF: The role of small non-coding RNAs in genome stability and chromatin organization. J Cell Sci 2010, 123:1825-1839.

38. Henikoff S, Ahmad K, Malik HS: The centromere paradox: stable inheritance with rapidly evolving DNA. Science 2001, 293:1098-1102.

39. Smith KM, Galazka JM, Phatale PA, Connolly LR, Freitag M: Centromeres of filamentous fungi. Chromosome Res 2012, 20:635-656.

40. Smith KM, Phatale PA, Sullivan CM, Pomraning KR, Freitag M: Heterochromatin is required for normal distribution of Neurospora crassa CenH3. Mol Cell Biol 2011, 31:2528-2542.

41. Reinhart BJ, Bartel DP: Small RNAs correspond to centromere heterochromatic repeats. Science 2002, 297:1831.

42. Pidoux AL, Allshire RC: The role of heterochromatin in centromere function. Philos Trans $R$ Soc Lond B Biol Sci 2005, 360:569-579.

43. Fishel B, Amstutz H, Baum M, Carbon J, Clarke L: Structural organization and functional analysis of centromeric DNA in the fission yeast Schizosaccharomyces pombe. Mol Cell Biol 1988, 8:754-763.

44. Allshire RC, Nimmo ER, Ekwall K, Javerzat JP, Cranston G: Mutations derepressing silent centromeric domains in fission yeast disrupt chromosome segregation. Genes Dev 1995, 9:218-233.

45. Allshire RC, Javerzat JP, Redhead NJ, Cranston G: Position Effect Variegation at Fission Yeast Centromeres. Cell 1994, 76:157-169.

46. Yadav V, Yang F, Reza MH, Liu S, Valent B, Sanyal K, Naqvi NI: Cellular Dynamics and Genomic Identity of Centromeres in Cereal Blast Fungus. MBio 2019, 10.

47. Weiberg A, Wang M, Bellinger M, Jin H: Small RNAs: a new paradigm in plant-microbe interactions. Annu Rev Phytopathol 2014, 52:495-516.

48. Weiberg A, Jin H: Small RNAs--the secret agents in the plant-pathogen interactions. Curr Opin Plant Biol 2015, 26:87-94.

49. Weiberg A, Wang M, Lin FM, Zhao H, Zhang Z, Kaloshian I, Huang HD, Jin H: Fungal small RNAs suppress plant immunity by hijacking host RNA interference pathways. Science 2013, 342:118-123.

50. Cai Q, Qiao L, Wang M, He B, Lin FM, Palmquist J, Huang HD, Jin H: Plants send small RNAs in extracellular vesicles to fungal pathogen to silence virulence genes. Science 2018. 
51. Wang B, Sun $\mathrm{Y}$, Song N, Zhao M, Liu R, Feng H, Wang X, Kang Z: Puccinia striiformis f. sp. tritici microRNAlike RNA 1 (Pst-milR1), an important pathogenicity factor of Pst, impairs wheat resistance to Pst by suppressing the wheat pathogenesis-related 2 gene. New Phytol 2017, 215:338-350.

52. Derbyshire $M$, Mbengue $M$, Barascud $M$, Navaud O, Raffaele S: Small RNAs from the plant pathogenic fungus Sclerotinia sclerotiorum highlight host candidate genes associated with quantitative disease resistance. Mol Plant Pathol 2019, 20:1279-1297.

53. Axtell MJ, Westholm JO, Lai EC: Vive la difference: biogenesis and evolution of microRNAs in plants and animals. Genome Biol 2011, 12:221.

54. Dai X, Zhuang Z, Zhao PX: psRNATarget: a plant small RNA target analysis server (2017 release). Nucleic Acids Res 2018.

55. Shahid S, Kim G, Johnson NR, Wafula E, Wang F, Coruh C, Bernal-Galeano V, Phifer T, dePamphilis CW, Westwood JH, Axtell MJ: MicroRNAs from the parasitic plant Cuscuta campestris target host messenger RNAs. Nature 2018, 553:82-85.

56. Servant N, Varoquaux N, Lajoie BR, Viara E, Chen CJ, Vert JP, Heard E, Dekker J, Barillot E: HiC-Pro: an optimized and flexible pipeline for Hi-C data processing. Genome Biol 2015, 16:259.

57. Ramirez F, Bhardwaj V, Arrigoni L, Lam KC, Gruning BA, Villaveces J, Habermann B, Akhtar A, Manke T: Highresolution TADs reveal DNA sequences underlying genome organization in flies. Nat Commun 2018, 9:189.

58. Cabanettes F, Klopp C: D-GENIES: dot plot large genomes in an interactive, efficient and simple way. PeerJ 2018, 6:e4958.

59. Li H: Minimap2: pairwise alignment for nucleotide sequences. Bioinformatics 2018, 34:3094-3100.

60. Ondov BD, Treangen TJ, Melsted P, Mallonee AB, Bergman NH, Koren S, Phillippy AM: Mash: fast genome and metagenome distance estimation using MinHash. Genome Biol 2016, 17:132.

61. Upadhyaya NM, Garnica DP, Karaoglu H, Sperschneider J, Nemri A, Xu B, Mago R, Cuomo CA, Rathjen JP, Park RF, et al: Comparative genomics of Australian isolates of the wheat stem rust pathogen Puccinia graminis f. sp. tritici reveals extensive polymorphism in candidate effector genes. Front Plant Sci 2014 , 5:759.

62. Soneson C, Love MI, Robinson MD: Differential analyses for RNA-seq: transcript-level estimates improve gene-level inferences. F1000Res 2015, 4:1521.

63. Love MI, Huber W, Anders S: Moderated estimation of fold change and dispersion for RNA-seq data with DESeq2. Genome Biol 2014, 15:550.

64. Gotz S, Garcia-Gomez JM, Terol J, Williams TD, Nagaraj SH, Nueda MJ, Robles M, Talon M, Dopazo J, Conesa A: High-throughput functional annotation and data mining with the Blast2GO suite. Nucleic Acids Res 2008, 36:3420-3435.

65. Kim D, Langmead B, Salzberg SL: HISAT: a fast spliced aligner with low memory requirements. Nat Methods 2015, 12:357-360.

66. Schwessinger B, Chen YJ, Tien R, Vogt JK, Sperschneider J, Nagar R, McMullan M, Sicheritz-Ponten T, Sorensen CK, Hovmoller MS, et al: Distinct Life Histories Impact Dikaryotic Genome Evolution in the Rust Fungus Puccinia striiformis Causing Stripe Rust in Wheat. Genome Biol Evol 2020, 12:597-617.

67. Schwessinger B, Sperschneider J, Cuddy WS, Garnica DP, Miller ME, Taylor JM, Dodds PN, Figueroa M, Park RF, Rathjen JP: A near-complete haplotype-phased genome of the dikaryotic wheat stripe rust fungus Puccinia striiformis f. sp. tritici reveals high interhaplotype diversity. mBio 2018, 9.

68. Quesneville H, Bergman CM, Andrieu O, Autard D, Nouaud D, Ashburner M, Anxolabehere D: Combined evidence annotation of transposable elements in genome sequences. PLoS Comput Biol 2005, 1:166-175.

69. Flutre T, Duprat E, Feuillet C, Quesneville H: Considering Transposable Element Diversification in De Novo Annotation Approaches. Plos One 2011, 6.

70. Bao WD, Kojima KK, Kohany O: Repbase Update, a database of repetitive elements in eukaryotic genomes. Mobile DNA 2015, 6.

71. De Coster W, D'Hert S, Schultz DT, Cruts M, Van Broeckhoven C: NanoPack: visualizing and processing longread sequencing data. Bioinformatics 2018, 34:2666-2669.

72. Stoiber M, Quick J, Egan R, Eun Lee J, Celniker S, Neely RK, Loman N, Pennacchio LA, Brown J: De novo Identification of DNA Modifications Enabled by Genome-Guided Nanopore Signal Processing. bioRxiv 2017:094672.

73. Rice P, Longden I, Bleasby A: EMBOSS: the European Molecular Biology Open Software Suite. Trends Genet 2000, 16:276-277.

74. Martin M: Cutadapt removes adapter sequences from high-throughput sequencing reads. $20112011,17$. 
75. Consortium TR: RNAcentral: a comprehensive database of non-coding RNA sequences. Nucleic Acids Res 2016.

76. Nawrocki EP, Burge SW, Bateman A, Daub J, Eberhardt RY, Eddy SR, Floden EW, Gardner PP, Jones TA, Tate J, Finn RD: Rfam 12.0: updates to the RNA families database. Nucleic Acids Res 2015, 43:D130-137.

77. Stocks MB, Moxon S, Mapleson D, Woolfenden HC, Mohorianu I, Folkes L, Schwach F, Dalmay T, Moulton V: The UEA SRNA workbench: a suite of tools for analysing and visualizing next generation sequencing microRNA and small RNA datasets. Bioinformatics 2012, 28:2059-2061.

78. Langmead B, Trapnell C, Pop M, Salzberg SL: Ultrafast and memory-efficient alignment of short DNA sequences to the human genome. Genome Biol 2009, 10:R25.

79. International Wheat Genome Sequencing C, investigators IRp, Appels R, Eversole K, Feuillet C, Keller B, Rogers J, Stein N, investigators Iw-gap, Pozniak $\mathrm{CJ}$, et al: Shifting the limits in wheat research and breeding using a fully annotated reference genome. Science 2018, 361.

80. Axtell MJ: ShortStack: comprehensive annotation and quantification of small RNA genes. RNA 2013, 19:740-751.

81. Robinson MD, McCarthy DJ, Smyth GK: edgeR: a Bioconductor package for differential expression analysis of digital gene expression data. Bioinformatics 2010, 26:139-140.

82. Krzywinski M, Schein J, Birol I, Connors J, Gascoyne R, Horsman D, Jones SJ, Marra MA: Circos: an information aesthetic for comparative genomics. Genome Res 2009, 19:1639-1645.

83. Quinlan AR, Hall IM: BEDTools: a flexible suite of utilities for comparing genomic features. Bioinformatics 2010, 26:841-842.

84. Edgar RC: MUSCLE: multiple sequence alignment with high accuracy and high throughput. Nucleic Acids Res 2004, 32:1792-1797.

85. Price MN, Dehal PS, Arkin AP: FastTree 2--approximately maximum-likelihood trees for large alignments. PLoS One 2010, 5:e9490.

86. Huerta-Cepas J, Serra F, Bork P: ETE 3: Reconstruction, Analysis, and Visualization of Phylogenomic Data. Mol Biol Evol 2016, 33:1635-1638. 